\title{
Differenze di genere in matematica: dagli studi internazionali alla situazione italiana
}

\section{Gender differences in mathematics: from the international literature to the Italian situation}

\author{
Chiara Giberti \\ Facoltà di Scienze della Formazione - Libera Università di Bolzano, Italia
}

Sunto / Dalle principali rilevazioni nazionali e internazionali emerge una forte disparità nei risultati di maschi e femmine nelle prove di matematica: gli studenti ottengono risultati migliori delle studentesse nella maggior parte delle nazioni e a tutti i livelli scolastici. Nel panorama internazionale, l'Italia risulta essere uno dei Paesi in cui i risultati relativi alle differenze di genere in matematica sono più allarmanti e i dati delle prove INVALSI confermano un gender gap molto marcato a favore dei maschi, presente a tutti i livelli scolastici. Questo articolo presenta la situazione internazionale relativa alle differenze di genere in matematica a partire dalle prove standardizzate, prestando particolare attenzione alla situazione italiana; quindi viene presentato un excursus dettagliato sulle cause alla base del gender gap in matematica, analizzando i principali studi sull'argomento e cercando di fornire una chiave di lettura completa, seppur complessa, di questo fenomeno.

Parole Chiave: differenze di genere; prove standardizzate; INVALSI; STEM.

\begin{abstract}
National and international surveys highlight a strong disparity in male and female results in mathematics: boys outperform girls in almost every country at all scholastic levels. In the international context, Italy appears to be one of the countries where the results related to gender differences in mathematics are more alarming and INVALSI test data confirm a very marked gender gap in favour of males at all scholastic levels. In this paper we present the international situation regarding gender differences in mathematics, starting from large-scale assessment and paying particular attention to the Italian context. We then present a detailed overview of the causes underlying the gender gap in mathematics, analysing the main studies on this issue and attempting to provide a complete, though complex, interpretation of this phenomenon.
\end{abstract}

Keywords: gender gap; large scale assessment; INVALSI; STEM.

\section{Introduzione}

II numero di donne impegnate in materie STEM (acronimo di: Science, Technology, Engineering and Mathematics) negli ultimi anni risulta essere in aumento; nonostante ciò quantitativamente permane un forte squilibrio a favore della componente maschile sia nel numero di iscrizioni all'università in corsi scientifici, sia nelle posizioni lavorative legate alle STEM (Hill, Corbett \& St. Rose, 2010; OECD, 2015).

Alcuni fattori che hanno portato a questa disparità di numero tra uomini e donne impegnati nelle materie scientifiche possono essere ricercati, in primo luogo, nella storia del costume e della società (MacKinnon, 1990; Leder \& Forgasz, 2008). Storicamente, infatti, I'accesso alla cultura scientifica e, in particolare, lo studio della matematica era riservato quasi esclusivamente agli uomini e ben pochi nomi femminili vengono ricordati nella storia della matematica prima del secolo scorso (MacKinnon, 1990; Leder \& Forgasz, 2008). 
Dall'inizio del 1900, sono stati fatti grandi passi avanti per permettere alle donne uguali diritti di accesso all'istruzione e al mondo del lavoro e per combattere gli stereotipi legati al genere. Dagli anni ' 70 , in molti Paesi sono state introdotte politiche educative finalizzate a incoraggiare le ragazze a studiare le scienze e la matematica e a garantire loro le stesse possibilità degli uomini anche nel mondo del lavoro (Leder \& Forgasz, 2008); nonostante ciò differenze di genere nelle materie STEM sussistono ancora oggi (Hill et al., 2010).

Nel settore delle materie STEM la matematica risulta avere un ruolo fondamentale per lo sviluppo e la conoscenza anche delle altre discipline (Hill et al., 2010) e, per questo motivo, molti studi si sono focalizzati proprio sulle differenze di genere in matematica.

Negli ultimi anni, il tema delle differenze di genere è stato oggetto di numerosi studi condotti da diversi punti di vista. Sono numerose infatti anche le teorie che non rientrano nella ricerca in didattica ma che appartengono piuttosto ad altri settori quali la sociologia e la psicologia (Leder, 1992; Byrnes, 2005; Pajares, 2005). La psicologia, negli ultimi anni, ha sviluppato un ampio settore di ricerca proprio legato alle difficoltà di apprendimento della matematica da parte delle ragazze legando i minori risultati a fattori di tipo affettivo e psicologico come l'ansia e la sicurezza in se stessi (e.g. Primi, Busdraghi, Tomasetto, Morsanyi \& Chiesi, 2014; Lindberg, Hyde, Petersen \& Linn, 2010).

L'introduzione, a livello internazionale, di prove standardizzate volte a monitorare l'apprendimento della matematica e di altre discipline, ha permesso di studiare le differenti performance di maschi e femmine anche confrontando Paesi con culture e sistemi di istruzione molto diversi. In particolare, i risultati delle prove OECD PISA e IEA TIMSS hanno messo, negli ultimi anni, in grande risalto le differenze di genere e hanno permesso di studiarle attraverso la comparazione tra diverse aree geografiche e in relazione agli indici di status socio-economico e culturale.

Questo articolo si propone di fare un excursus delle ricerche sulle differenze di genere in matematica: la prima parte sarà dedicata all'analisi dei principali risultati delle prove standardizzate, mentre nei paragrafi successivi si andranno a ricercare le cause delle differenze emerse: saranno prese in considerazione soprattutto le ricerche provenienti dal settore della didattica della matematica, affiancate anche dai principali studi sul tema provenienti da altri settori.

\section{2}

\section{Metodologia}

In questo articolo, le principali ricerche in didattica della matematica sul tema delle differenze di genere saranno confrontate tra loro e integrate con studi provenienti da altri settori, in modo da fornire una visione complessiva di questo fenomeno.

Sono stati quindi presi in considerazione i principali articoli che trattano il tema delle differenze di genere in matematica presenti nelle riviste di classe A di didattica della matematica, secondo la classificazione (Törner \& Arzarello, 2013) prodotta congiuntamente dai membri dell'ERME (Executive Committee of the European Society for Research in Mathematics Education) e dell'EMS (Education Committee of the European Mathematical Society). Molti di questi studi si riferiscono direttamente ai risultati delle rilevazioni su larga scala IEA TIMSS e OECD PISA e per questo motivo si 
è deciso di presentare, innanzi tutto, i risultati di tali prove in termini di differenze di genere in matematica e commentare, in un secondo momento, tali risultati alla luce degli studi sopra descritti. Questa prima analisi permette di individuare le caratteristiche principali di questo fenomeno e, quando possibile in base ai risultati forniti dalle rilevazioni internazionali, di declinarle nelle diverse realtà nazionali.

A partire dalla selezione di articoli appartenenti al settore della didattica della matematica, la letteratura è stata ampliata sia approfondendo le ricerche citate in questi articoli, sia attraverso una ricerca indipendente dell'autrice al fine di comprendere meglio alcune delle evidenze emerse, anche analizzando studi provenienti da altri settori e riviste. Le caratteristiche così evidenziate relative alle differenze di genere in matematica possono essere prese in considerazione per fare uno stato dell'arte delle ricerche sul tema e possono fornire informazioni importanti per confrontare la letteratura relativa.

\section{Studio delle differenze di genere attraverso le prove standardizzate}

Leder e Forgasz nel 2008 forniscono una panoramica degli studi relativi alle differenze di genere nell'ambito della didattica della matematica e sottolineano l'importanza dell'uso delle prove standardizzate in questa direzione:

«I risultati dei test internazionali su larga scala, incluso il Programma per la Valutazione Internazionale dell'Allievo (Programme for International Student Assessment - PISA), hanno attirato notevolmente l'attenzione della comunità dei ricercatori in didattica della matematica e anche di tutti coloro che hanno un particolare interesse per le differenze di genere nell'apprendimento della matematica».

(Leder \& Forgasz, 2008, p. 516, traduzione dell'autore)

\subsection{Rilevazioni internazionali OECD PISA e IEA TIMSS}

Le principali indagini internazionali volte a misurare gli apprendimenti della matematica e di altre discipline sono le prove PISA promosse dall'Organisation for Economic Co-operation and Development (OECD) e quelle TIMSS promosse dall'International Association for Evaluation of Educational Achievement (IEA).

Le principali caratteristiche e differenze dei due studi sono riportate nella tabella alla pagina successiva (particolarmente interessante è l'anno 2015, I'ultimo, ad ora, nel quale si sono svolte contemporaneamente le due rilevazioni): 
Tabella 1

Principali caratteristiche delle prove OECD PISA e IEA TIMSS

\begin{tabular}{|c|c|c|c|}
\hline Prova & OECD PISA & IEA TIMSS & \\
\hline Prima edizione e cadenza & 2000; cadenza triennale & 1995; cadenza quadriennale & \\
\hline Ambiti di investigazione & $\begin{array}{l}\text { Lettura, Matematica, Scienze e } \\
\text { Financial literacy (ogni edizione } \\
\text { si focalizza in particolare su un } \\
\text { ambito) }\end{array}$ & Scienze e Matematica & \\
\hline Campione di riferimento & $\begin{array}{l}\text { Studenti quindicenni } \\
\text { scolarizzati (prove campionarie) }\end{array}$ & $\begin{array}{l}\text { Studenti frequentanti il grado } 4 \\
\text { e il grado } 8 \text { di scolarizzazione } \\
\text { corrispondenti alla quarta classe } \\
\text { di scuola elementare e alla terza } \\
\text { media (prove campionarie) }\end{array}$ & $\overline{1}$ \\
\hline Finalità & $\begin{array}{l}\text { Valutare l'acquisizione delle } \\
\text { competenze chiave per la piena } \\
\text { partecipazione alla società } \\
\text { moderna } 2\end{array}$ & $\begin{array}{l}\text { Valutare l'acquisizione dei } \\
\text { contenuti e delle abilità } \\
\text { curricolari da parte degli } \\
\text { studenti }^{3}\end{array}$ & $2-3$ \\
\hline Partecipanti 2015 & $\begin{array}{l}72 \text { Paesi per un totale di circa } \\
540000 \text { studenti coinvolti (di } \\
\text { queste } 35 \text { sono nazioni OECD) }\end{array}$ & $\begin{array}{l}57 \text { Paesi (non tutti compresi in } \\
\text { quelli partecipanti a OECD PISA) }\end{array}$ & \\
\hline
\end{tabular}

\subsection{Le differenze di genere nei risultati delle rilevazioni internazionali}

L'uso delle prove standardizzate per la ricerca in didattica della matematica è aumentato negli ultimi anni, anche se le potenzialità che tali prove potrebbero avere non sono ancora pienamente sfruttate (Maffia \& Giberti, 2016; Sfard, 2005). I risultati riportati nei prossimi paragrafi, sono tratti dai report delle prove TIMSS e PISA e provengono principalmente dalle rilevazioni del 2015 che, come già specificato, è un anno particolarmente interessante in quanto sono state svolte in concomitanza entrambe le rilevazioni.

\subsubsection{Risultati dell'indagine PISA 2015}

Nel 2015 le nazioni OECD che hanno partecipato alle rilevazioni PISA di matematica sono state 35 e il punteggio medio ottenuto nella prova da queste nazioni è stato di 490 punti (per approfondire il modo in cui vengono attribuiti i punteggi nelle prove, a seguito dell'ancoraggio, si rimanda al rapporto tecnico: OECD, 2016d). Nel grafico seguente (Figura 1) sono riportati, nella seconda colonna evidenziata in azzurro, i punteggi medi ottenuti nella prova di matematica per ogni nazione partecipante e,

\footnotetext{
1. In Italia la scuola elementare viene detta scuola primaria, la scuola media, scuola secondaria di primo grado, la scuola medio-superiore viene detta scuola secondaria di secondo grado.

2. La definizione di mathematical licteracy assunta è la seguente (OECD, 2016c, p. 66, traduzione dall'autore): La competenza matematica è la capacità di un individuo di formulare, utilizzare e interpretare la matematica in una varietà di contesti. Include ragionare in termini matematici e utilizzare concetti matematici, procedure, fatti e strumenti per descrivere, spiegare e predire fenomeni. La competenza matematica aiuta gli individui a riconoscere il ruolo che la matematica gioca nel mondo, a operare valutazioni e a prendere decisioni fondate che consentano loro di essere cittadini impegnati, riflessivi e con un ruolo costruttivo

3. I test somministrati agli studenti sono relativi alle parti comuni dei curricula dei diversi Paesi.
} 
a fianco, la differenza tra il punteggio medio ottenuto dagli studenti maschi e quello ottenuto dalle studentesse. L'istogramma mostra quindi un gap a favore dei maschi nel caso in cui la barra sia a destra della linea verticale e un gap a favore delle femmine nel caso in cui la barra si sviluppi a sinistra. I simboli, riportati anche in legenda, indicano inoltre le differenze di genere considerando esclusivamente i lowest-achieving students (che si posizionano al di sotto del decimo percentile) e gli highest-achieving students (che si posizionano al di sopra del novantesimo percentile). Nella figura i colori più scuri indicano una differenza statisticamente significativa, sia per quanto riguarda le barre del grafico sia per quanto riguarda i simboli relativi ai percentili.

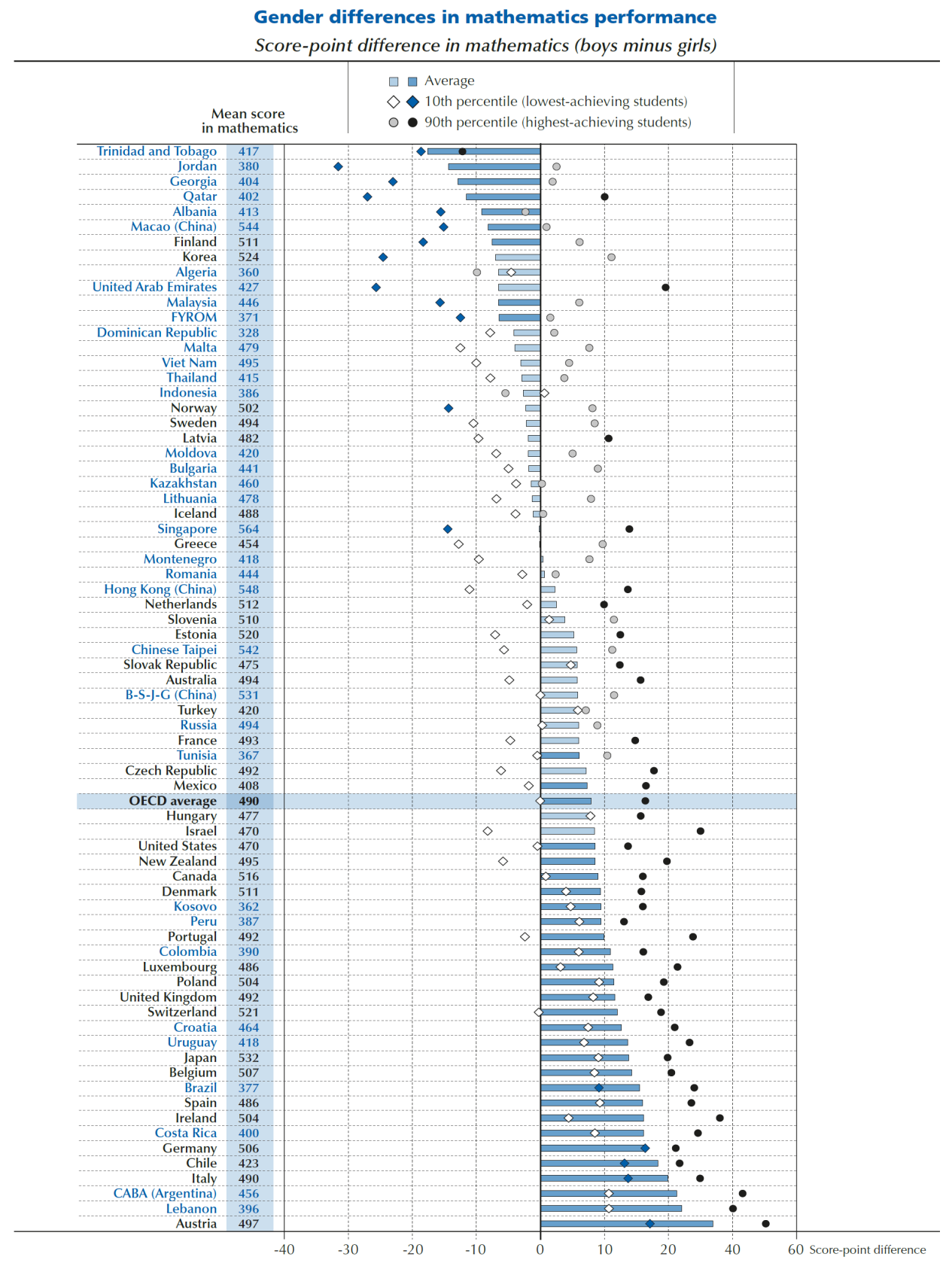

Se si considerano i risultati nel loro complesso, i maschi ottengono in media 8 punti in più rispetto alle coetanee (OECD, 2016a), le differenze però variano notevolmente di nazione in nazione. 
Le differenze di genere nella prova di matematica non si manifestano in modo uniforme in tutti i Paesi coinvolti nella rilevazione: circa metà delle nazioni presenta un gap a favore dei maschi che è statisticamente significativo, in quasi altrettanti Paesi il gap non è statisticamente significativo e addirittura in alcuni Paesi i risultati delle ragazze superano quelli dei ragazzi. La disomogeneità del fenomeno è anche una delle cause per cui alcuni studi non riscontrano un gap significativo: in base alle nazioni coinvolte nelle rilevazioni, infatti, si possono riscontrare maggiori o minori differenze di genere. Inoltre queste differenze non sembrano strettamente legate al punteggio complessivo ottenuto nella prova: tra i Paesi che mostrano un gender gap molto marcato si possono individuare sia nazioni con punteggi superiori alla media OECD (e.g. Giappone) sia nazioni che ottengono risultati nettamente inferiori alla media OECD (e.g. Brasile) e lo stesso avviene per i Paesi in cui il gender gap non è significativo o è addirittura a favore delle ragazze.

Le prove PISA coinvolgono gli studenti quindicenni che frequentano la scuola e, come specificato anche nei report PISA (OECD, 2016c), il fatto di non raggiungere tutti i quindicenni ma solo quelli scolarizzati e le difficoltà che in alcuni Paesi si riscontrano nel raggiungere alcune regioni per effettuare le somministrazioni inficiano la rappresentatività del campione. Per questo motivo nell'interpretare i grafici sopra bisogna anche tenere conto del fatto che in alcuni Paesi la copertura della popolazione potrebbe non essere sufficiente e che il campione degli studenti coinvolti potrebbe essere rappresentativo dei quindicenni scolarizzati e non di tutti i quindicenni della popolazione (per tali informazioni specifiche di ogni Paese si rimanda alla tabella 11.11 del rapporto tecnico PISA 2015: OECD, 2016d). In alcuni Paesi, in cui solo una minoranza delle donne può avere accesso all'istruzione e proseguire gli studi fino ai 15 anni, questi fattori possono influenzare notevolmente la misura del gender gap in matematica.

Inoltre, come già messo in luce dalle rilevazioni precedenti, il gap tra maschi e femmine risulta più marcato se si considerano solo gli highest-achieving students. In questo caso, il gap medio nei Paesi oggetto di indagine è di 16 punti e, per tutti i Paesi in cui il gap è significativo e a favore dei maschi, la differenza di performance (indicata con il cerchio) tra gli studenti che superano la soglia del novantesimo percentile risulta essere sempre significativa (cerchio nero) e superiore al gap relativo all'intera nazione (OECD, 2016a).

Per quanto riguarda il contesto italiano, sebbene gli studenti ottengano un punteggio medio complessivo pari alla media OECD (Figura 1), si osserva un notevole divario, pari a 20 punti, tra le performance dei ragazzi e le performance delle ragazze (OECD, 2016b). L'Italia è quindi uno dei Paesi OECD in cui le ragazze sono maggiormente svantaggiate in matematica e dal 2012 al 2015 il gap risulta essere invariato se non leggermente aumentato mentre nella maggior parte dei Paesi il gap negli stessi anni è invariato o leggermente diminuito (OECD, 2015).

\subsubsection{Risultati dell'indagine TIMSS 2015}

Le osservazioni appena proposte vengono in buona parte confermate anche dai risultati delle prove TIMSS 2015 che indagano i livelli scolastici 4 e 8, corrispondenti alla classe quarta scuola elementare e alla classe terza di scuola media nel sistema scolastico italiano.

Nelle prove TIMSS la scala dei punteggi viene centrata sul 500 che corrisponde quindi al punteggio medio della prova considerando tutte le nazioni che partecipano all'indagine; le unità della scala vengono scelte in modo che 100 punti corrispondano alla de- 
viazione standard della distribuzione dei punteggi (Mullis, Martin, Foy \& Hooper, 2016). Nei grafici seguenti sono riportati i risultati delle prove TIMSS del 2015 somministrate per il grado 4 (Figura 2) e per il grado 8 (Figura 3). Per ogni nazione coinvolta nella rilevazione, vengono riportati il punteggio medio ottenuto dai maschi e dalle femmine, insieme alla percentuale di maschi e femmine presente nel campione. La differenza tra il punteggio medio ottenuto dai maschi e quello ottenuto dalle femmine viene riportato sia in termini numerici, sia attraverso l'istogramma nell'ultima colonna.

Anche in questi grafici, le differenze statisticamente significative vengono indicate in blu, mentre quando il gap non è significativo la barra dell'istogramma è grigia.

In calce ai grafici, è riportata la spiegazione dei simboli inseriti a fianco delle singole nazioni che indicano specificità legate alla rappresentatività del campione e le linee guida utilizzate da TIMSS per la creazione di un campione nazionale. Le informazioni relative al gender gap che possono essere estrapolate dai grafici devono essere lette, anche in questo caso, tenendo conto anche della rappresentatività del campione rispetto alla intera popolazione.

\begin{tabular}{|c|c|c|c|c|c|c|c|c|c|c|}
\hline \multirow[b]{2}{*}{ Country } & \multicolumn{2}{|c|}{ Girls } & \multicolumn{2}{|c|}{ Boys } & \multirow{2}{*}{$\begin{array}{c}\text { Difference } \\
\text { (Absolute } \\
\text { Value) }\end{array}$} & \multicolumn{5}{|c|}{ Gender Difference } \\
\hline & $\begin{array}{l}\text { Percent of } \\
\text { Students }\end{array}$ & $\begin{array}{c}\text { Average Scale } \\
\text { Score }\end{array}$ & $\begin{array}{l}\text { Percent of } \\
\text { Students }\end{array}$ & $\begin{array}{c}\text { Average Scale } \\
\text { Score }\end{array}$ & & & $\begin{array}{c}\text { Girls } \\
\text { ored Highe }\end{array}$ & & $\begin{array}{c}\text { Boys } \\
\text { Scored Hight }\end{array}$ & \\
\hline$\psi$ Saudi Arabia & $49(1.0)$ & $405(4.4)$ & $51(1.0)$ & $363(6.5)$ & $43(7.7)$ & & & 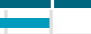 & & \\
\hline Oman & $50(0.7)$ & $436(3.0)$ & $50(0.7)$ & $415(2.8)$ & $22(2.9)$ & & & $\square$ & & \\
\hline Jordan & $46(2.4)$ & $399(3.3)$ & $54(2.4)$ & $379(4.9)$ & $20(5.8)$ & & & - & & \\
\hline South Africa (5) & $48(0.8)$ & $384(3.8)$ & $52(0.8)$ & $368(4.4)$ & $15(4.2)$ & & & E & & \\
\hline 2 Bahrain & $50(0.7)$ & $459(1.7)$ & $50(0.7)$ & $443(2.3)$ & $15(2.5)$ & & & E & & \\
\hline$\psi$ Kuwait & $51(2.0)$ & $359(5.4)$ & $49(2.0)$ & $347(5.6)$ & $12(6.2)$ & & & 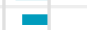 & & \\
\hline Iran, Islamic Rep. of & $50(0.9)$ & $437(4.5)$ & $50(0.9)$ & $426(4.5)$ & $10(6.3)$ & & & $\bar{\square}$ & & \\
\hline Indonesia & $48(0.6)$ & $403(4.0)$ & $52(0.6)$ & $393(3.9)$ & $10(2.7)$ & & & - & & \\
\hline Finland & $48(0.8)$ & $540(2.3)$ & $52(0.8)$ & $531(2.6)$ & $9(2.9)$ & & & - & & \\
\hline Bulgaria & $49(0.8)$ & $527(5.7)$ & $51(0.8)$ & $522(5.1)$ & $5(2.9)$ & & & 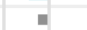 & & \\
\hline Norway (5) & $49(0.9)$ & $551(2.6)$ & $51(0.9)$ & $547(3.1)$ & $4(2.9)$ & & & - & & \\
\hline 2 Singapore & $48(0.5)$ & $620(3.9)$ & $52(0.5)$ & $616(4.3)$ & $4(3.0)$ & & & - & & \\
\hline United Arab Emirates & $48(2.2)$ & $453(3.9)$ & $52(2.2)$ & $450(3.4)$ & $3(5.4)$ & & & I & & \\
\hline 1 Georgia & $49(0.9)$ & $465(3.9)$ & $51(0.9)$ & $461(4.4)$ & $3(4.0)$ & & & 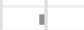 & & \\
\hline${ }^{3}$ Serbia & $48(0.8)$ & $520(3.7)$ & $52(0.8)$ & $517(4.7)$ & $3(4.7)$ & & & 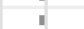 & & \\
\hline Qatar & $51(2.5)$ & $440(4.1)$ & $49(2.5)$ & $438(4.9)$ & $3(5.9)$ & & & - & & \\
\hline${ }^{2}$ Lithuania & $50(0.9)$ & $537(2.8)$ & $50(0.9)$ & $534(3.1)$ & $2(3.3)$ & & & I & & \\
\hline Kazakhstan & $49(0.8)$ & $546(4.6)$ & $51(0.8)$ & $543(4.8)$ & $2(2.8)$ & & & i & & \\
\hline Morocco & $48(0.7)$ & $378(3.5)$ & $52(0.7)$ & $377(3.9)$ & $1(2.8)$ & & & 1 & & \\
\hline 2 Sweden & $49(1.0)$ & $519(3.2)$ & $51(1.0)$ & $518(3.2)$ & $1(3.0)$ & & & 1 & & \\
\hline Russian Federation & $49(0.9)$ & $564(3.7)$ & $51(0.9)$ & $564(3.7)$ & $1(2.8)$ & & & I & & \\
\hline Japan & $50(0.5)$ & $593(2.0)$ & $50(0.5)$ & $593(2.5)$ & $0(2.3)$ & & & & & \\
\hline Chile & $49(1.7)$ & $458(2.8)$ & $51(1.7)$ & $459(3.0)$ & $1(3.2)$ & & & 1 & & \\
\hline Poland & $50(0.8)$ & $534(2.3)$ & $50(0.8)$ & $536(2.7)$ & $1(2.5)$ & & & 1 & & \\
\hline Turkey & $49(0.6)$ & $482(3.2)$ & $51(0.6)$ & $484(3.5)$ & $2(2.7)$ & & & I & & \\
\hline ₹ Northern Ireland & $50(1.1)$ & $569(3.8)$ & $50(1.1)$ & $571(3.1)$ & $2(3.8)$ & & & 1 & & \\
\hline New Zealand & $49(0.7)$ & $489(2.8)$ & $51(0.7)$ & $492(2.6)$ & $2(2.8)$ & & & I & & \\
\hline Germany & $48(0.7)$ & $520(2.4)$ & $52(0.7)$ & $523(2.3)$ & $3(2.3)$ & & & I & & \\
\hline Ireland & $47(1.5)$ & $545(2.6)$ & $53(1.5)$ & $549(2.9)$ & $4(3.4)$ & & & . & & \\
\hline Slovenia & $49(0.8)$ & $518(2.1)$ & $51(0.8)$ & $522(2.4)$ & $4(2.6)$ & & & I & & \\
\hline Chinese Taipei & $49(0.6)$ & $594(2.2)$ & $51(0.6)$ & $599(2.3)$ & $6(2.5)$ & & & 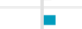 & & \\
\hline † Belgium (Flemish) & $50(0.9)$ & $543(2.4)$ & $50(0.9)$ & $549(2.4)$ & $6(2.4)$ & & & - & & \\
\hline Hungary & $49(0.9)$ & $526(3.4)$ & $51(0.9)$ & $532(3.8)$ & $6(3.4)$ & & & - & & \\
\hline France & $49(0.7)$ & $485(3.2)$ & $51(0.7)$ & $491(3.2)$ & $6(2.8)$ & & & - & & \\
\hline $2+$ Denmark & $49(0.8)$ & $536(3.1)$ & $51(0.8)$ & $542(3.0)$ & $6(2.8)$ & & & - & & \\
\hline England & $51(0.7)$ & $543(3.0)$ & $49(0.7)$ & $549(3.3)$ & $6(2.9)$ & & & - & & \\
\hline Cyprus & $49(0.7)$ & $520(2.9)$ & $51(0.7)$ & $526(3.1)$ & $6(2.7)$ & & & - & & \\
\hline $2+$ United States & $51(0.6)$ & $536(2.3)$ & $49(0.6)$ & $543(2.6)$ & $7(1.9)$ & & & - & & \\
\hline Czech Republic & $49(0.9)$ & $525(3.0)$ & $51(0.9)$ & $532(2.5)$ & $7(3.2)$ & & & 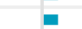 & & \\
\hline Korea, Rep. of & $48(0.5)$ & $604(2.3)$ & $52(0.5)$ & $612(2.5)$ & $7(1.9)$ & & & a & & \\
\hline † Netherlands & $50(0.9)$ & $526(1.8)$ & $50(0.9)$ & $534(2.2)$ & $8(2.2)$ & & & - & & \\
\hline Australia & $49(1.0)$ & $513(3.1)$ & $51(1.0)$ & $522(3.9)$ & $9(3.5)$ & & & E & & \\
\hline $12+$ Canada & $49(0.5)$ & $506(2.5)$ & $51(0.5)$ & $515(2.6)$ & $9(2.1)$ & & & a & & \\
\hline † Hong Kong SAR & $46(1.5)$ & $609(3.8)$ & $54(1.5)$ & $619(2.8)$ & $10(3.3)$ & & & - & & \\
\hline 2 Portugal & $49(0.8)$ & $536(2.4)$ & $51(0.8)$ & $547(2.5)$ & $11(2.2)$ & & & E & & \\
\hline Slovak Republic & $48(0.9)$ & $493(3.0)$ & $52(0.9)$ & $504(2.6)$ & $11(2.6)$ & & & - & & \\
\hline${ }^{2}$ Spain & $49(0.9)$ & $499(2.7)$ & $51(0.9)$ & $511(2.7)$ & $12(2.4)$ & & & E & & \\
\hline Croatia & $49(0.8)$ & $496(2.1)$ & $51(0.8)$ & $508(2.3)$ & $12(2.7)$ & & & E & & \\
\hline 2 Italy & $49(0.7)$ & $497(2.7)$ & $51(0.7)$ & $517(3.0)$ & $20(2.7)$ & & & $=$ & & \\
\hline International Avg. & $49(0.2)$ & $505(0.5)$ & $51(0.2)$ & $505(0.5)$ & & & & & & \\
\hline \multicolumn{6}{|l|}{ Benchmarking Participants } & & 40 & 0 & 40 & 80 \\
\hline${ }^{2} \Psi$ Abu Dhabi, UAE & $47(3.7)$ & $422(8.0)$ & $53(3.7)$ & $417(6.6)$ & $4(11.2)$ & & & -1 & & \\
\hline 1 Florida, US & $49(1.1)$ & $548(4.9)$ & $51(1.1)$ & $544(5.5)$ & $4(4.5)$ & & & 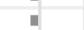 & & \\
\hline Dubai, UAE & $48(3.3)$ & $510(3.1)$ & $52(3.3)$ & $512(2.7)$ & $2(5.0)$ & & & i & & \\
\hline Norway (4) & $49(0.9)$ & $492(2.9)$ & $51(0.9)$ & $494(3.0)$ & $3(3.6)$ & & & I & & \\
\hline Buenos Aires, Argentina & $50(1.0)$ & $430(3.5)$ & $50(1.0)$ & $435(2.9)$ & $5(2.8)$ & & & - & & \\
\hline Ontario, Canada & $49(0.8)$ & $509(2.6)$ & $51(0.8)$ & $516(2.8)$ & $7(2.9)$ & & & - & & \\
\hline \$ Quebec, Canada & $50(1.0)$ & $531(3.9)$ & $50(1.0)$ & $541(4.8)$ & $11(3.8)$ & & & 믐 & & \\
\hline
\end{tabular}


Legenda:

Figura 2

Differenze di performance in base al genere nelle prove TIMSS 2015 di matematica - grado 4. Fonte: TIMSS 2015 Internationa/ Results in Mathematics (Mullis et al., 2016)

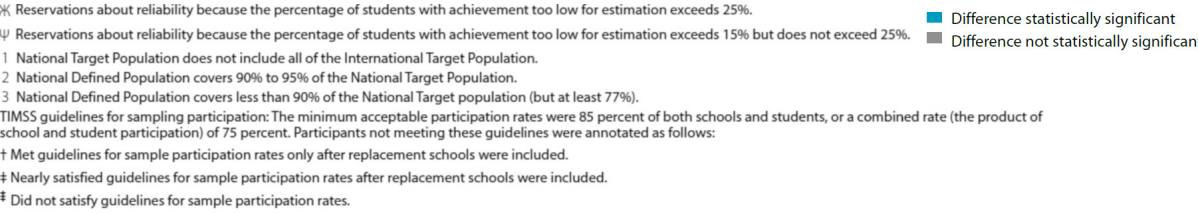

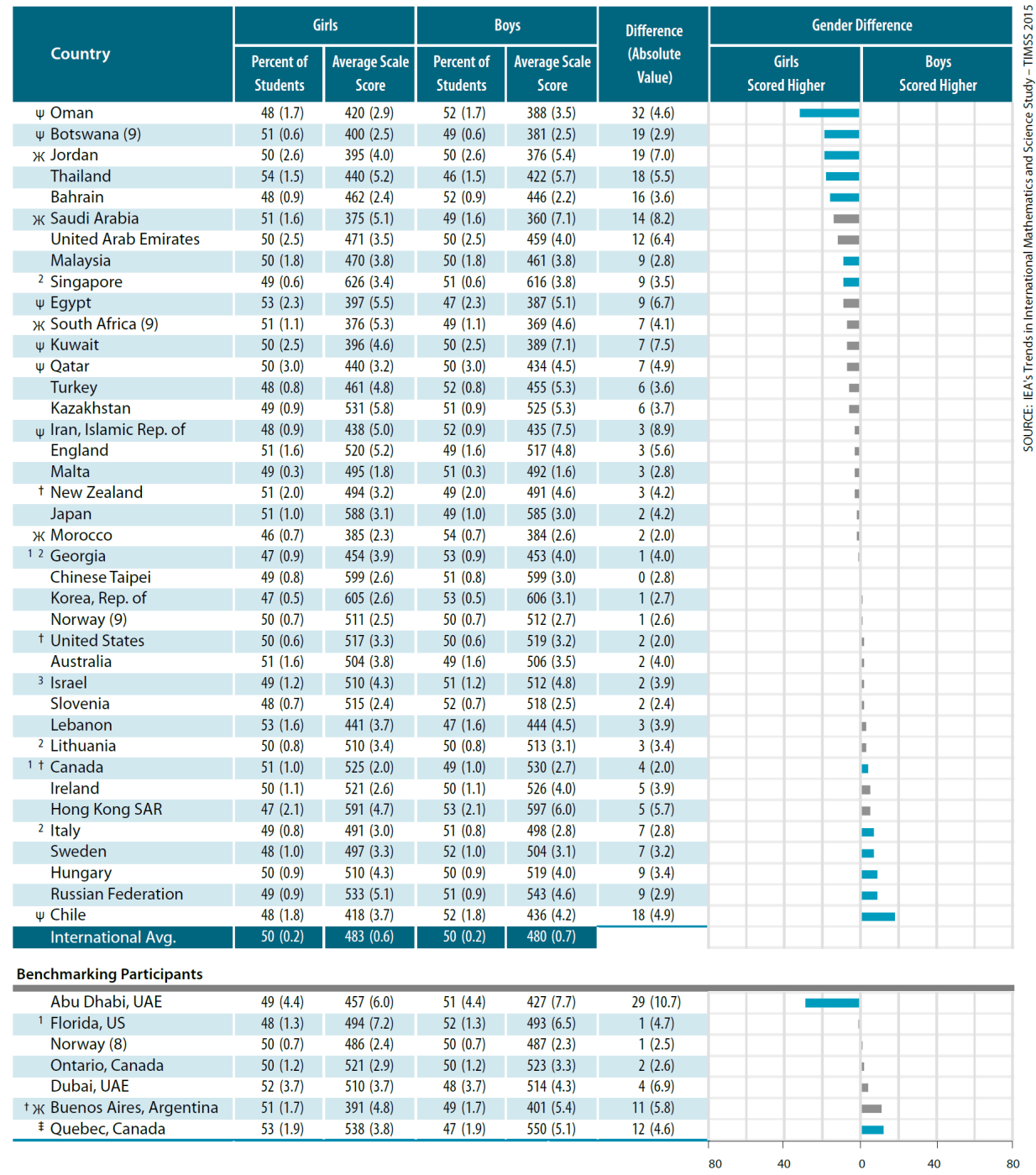

Legenda:

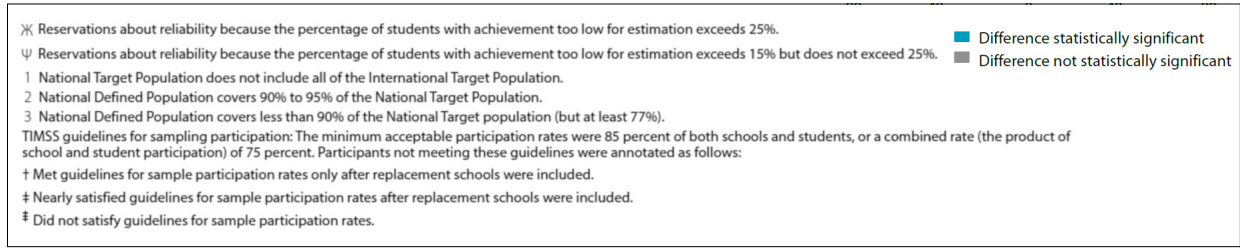

In entrambi i livelli, anche in questo caso, il gender gap non risulta uniformemente distribuito sulle diverse nazioni.

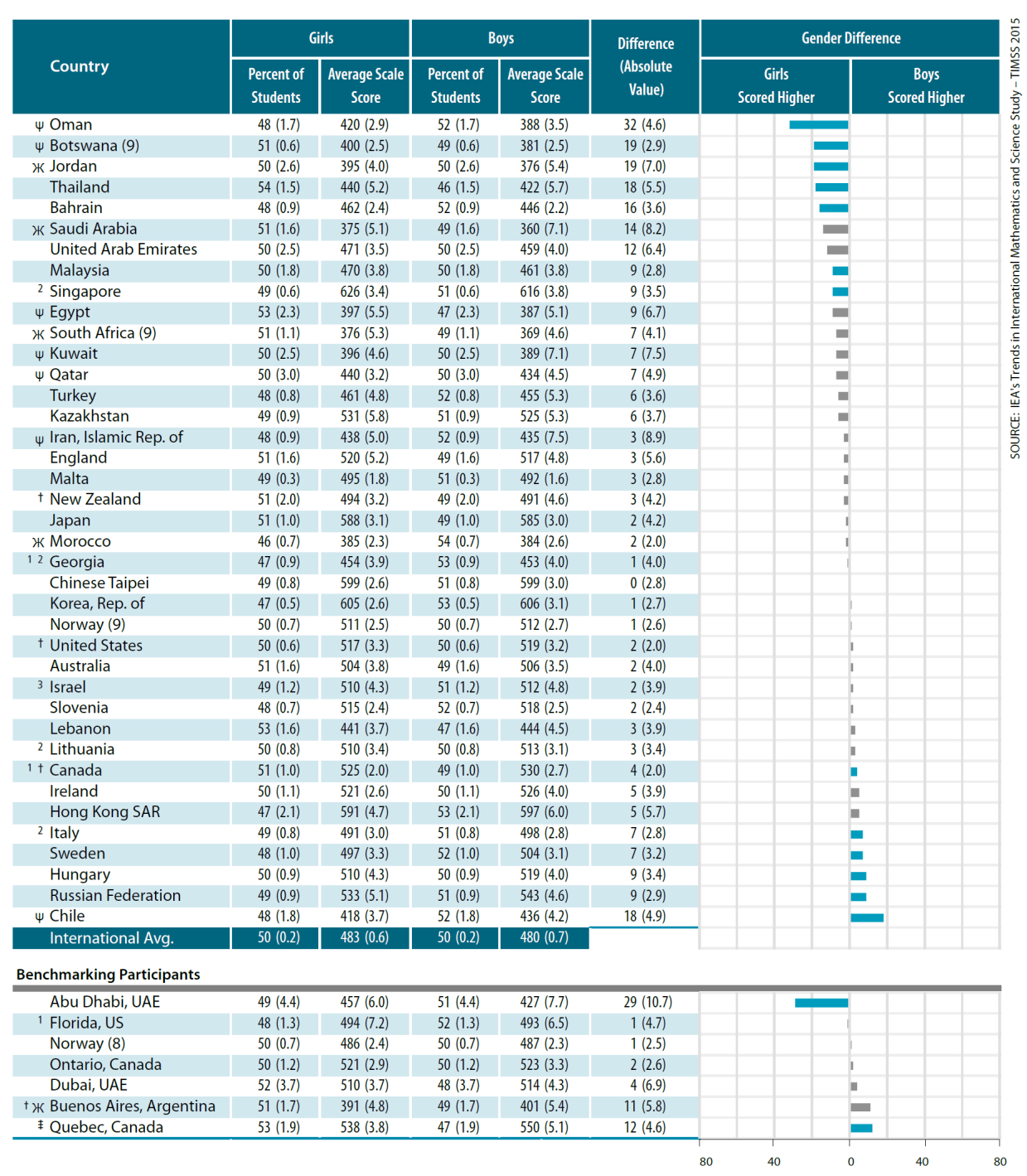

\section{Figura 3}

Differenze di performance in base al genere nelle prove TIMSS 2015 di matematica - grado 8 Fonte: TIMSS 2015 Inter-

national Results in Mathematics (Mullis et al., 2016) 
Le nazioni partecipanti nel 2015 per il grado 4 sono state 49 e in 18 di queste è presente una differenza tra i punteggi dei maschi e quelli delle femmine che risulta statisticamente significativa e, in media, di 9 punti a favore dei maschi. In 23 Paesi non si ha alcuna differenza significativa e solo in 8 le femmine ottengono risultati migliori. Nella prova del grado 8 , invece, le differenze di genere risultano generalmente meno marcate e in 26 nazioni su 39 non si ha alcun divario statisticamente significativo. Anche i risultati delle prove TIMSS 2015 confermano come, in termini di differenze di genere in matematica, la situazione dell'Italia sia ben delineata e critica. I risultati medi complessivi degli studenti italiani risultano, nelle prove di entrambi i livelli, vicini alla media dei Paesi coinvolti, ma si può notare che al grado 8 e ancor di più nella prova di grado 4, I'Italia mostra un forte divario di genere, uno dei più marcati in assoluto che è presente dai primi anni in cui sono state svolte le rilevazioni (Figura 4).

Figura 4

Evoluzione del gap di performance in base al genere nelle prove TIMSS

di matematica dal 2003 a 2015 in Italia. Fonte: TIMSS 2015 International Results in Mathematics (Mullis et al., 2016).

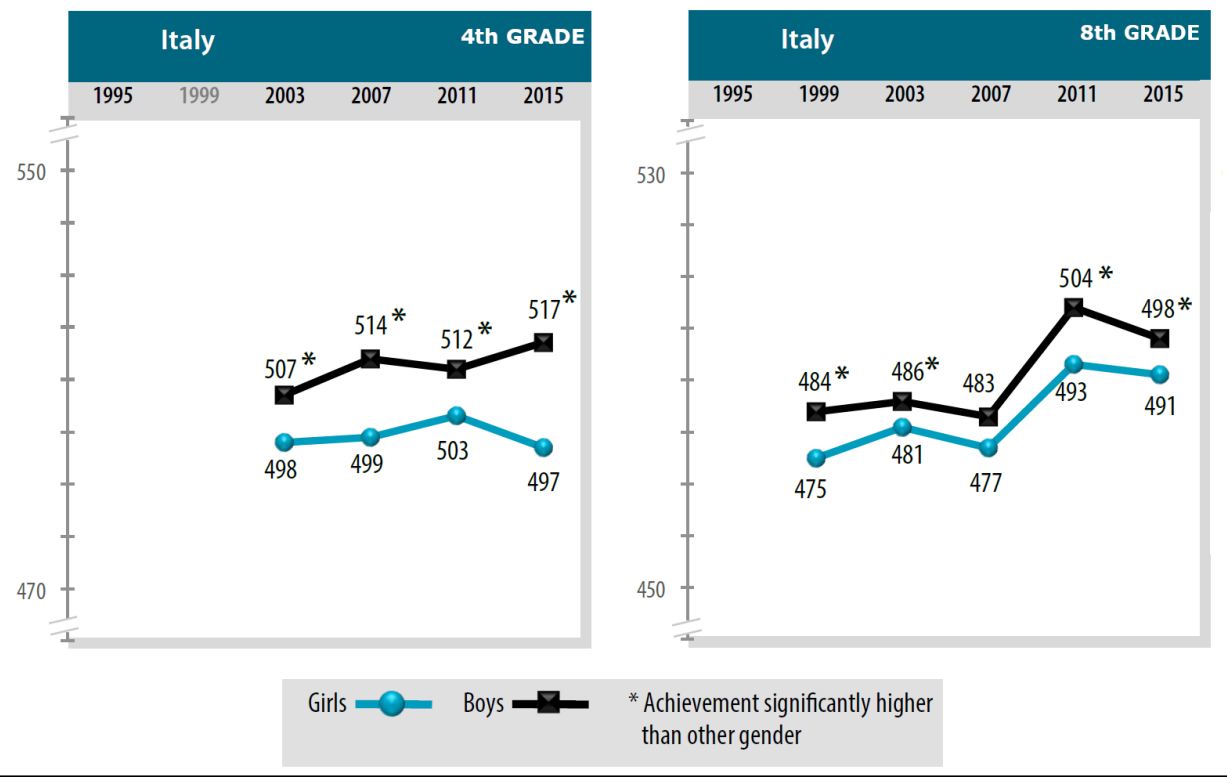

3.3 Differenze di genere in Italia: dalle prove internazionali alle prove INVALSI

Come si è visto nei paragrafi precedenti, i dati descrivono la situazione italiana come una delle più allarmanti all'interno dello scenario internazionale: I'Italia è una delle nazioni in cui il gender gap è maggiormente marcato e il divario di genere in matematica viene anche confermato dai test INVALSI effettuati a livello nazionale (INVALSI, 2016; Contini, Di Tommaso \& Mendolia, 2017).

In Italia dall'anno scolastico 2007/2008 sono state somministrate, in diversi livelli scolastici, prove standardizzate di matematica e italiano (grammatica e comprensione del testo) a cura del Servizio Nazionale di Valutazione (SNV) che fa capo all'Istituto INVALSI. L'obiettivo principale delle prove INVALSI è quello di valutare l'efficacia del sistema d'istruzione nazionale, attraverso il rilevamento di informazioni riguardanti gli apprendimenti degli studenti in matematica e italiano (dal 2018 la prova di terza media coinvolge anche l'inglese).

Le prove INVALSI sono dunque somministrate nelle scuole italiane da quasi un decennio e offrono un'enorme mole di dati relativi agli apprendimenti in italiano e in matematica degli studenti dalla scuola elementare alla scuola media. Nonostante ciò, I'uso di queste prove nel campo della ricerca in didattica della matematica risulta 
ancora limitato (Maffia \& Giberti, 2016) e, per quanto riguarda lo studio delle differenze di genere in matematica, questi dati vengono utilizzati quasi esclusivamente sul piano statistico: manca un'interpretazione in chiave didattica del fenomeno a livello nazionale. Nei Rapporti Nazionali INVALSI il gender gap viene solitamente presentato in termini di differenza tra il punteggio medio ottenuto dai maschi e quello ottenuto dalle femmine all'interno della prova.

Le ultime rilevazioni mostrano marcate differenze tra le performance di maschi e femmine in matematica e confermano quanto evidenziato dalle prove internazionali PISA e TIMSS.

II grafico seguente (Figura 5) riporta i risultati delle prove INVALSI di matematica svolte nel 2017, nei diversi livelli scolastici. Per ogni grado scolastico è riportato il punteggio medio ottenuto dalle femmine (in rosa) e il punteggio medio ottenuto dai maschi (in azzurro), con i relativi intervalli di confidenza. I risultati, in accordo con quelli delle precedenti rilevazioni INVALSI, mostrano un gap di genere statisticamente significativo in tutti i livelli scolastici coinvolti: seconda e quinta scuola elementare, terza scuola media e seconda scuola media superiore. ${ }^{4}$

Figura 5

Risultati di maschi e femmine nelle prove INVALSI di matematica del 2017

nei diversi livelli scolastici. Fonte: Rilevazione Nazionale degli apprendimenti 2016-17 - Rapporto Risultati (INVALSI, 2017).

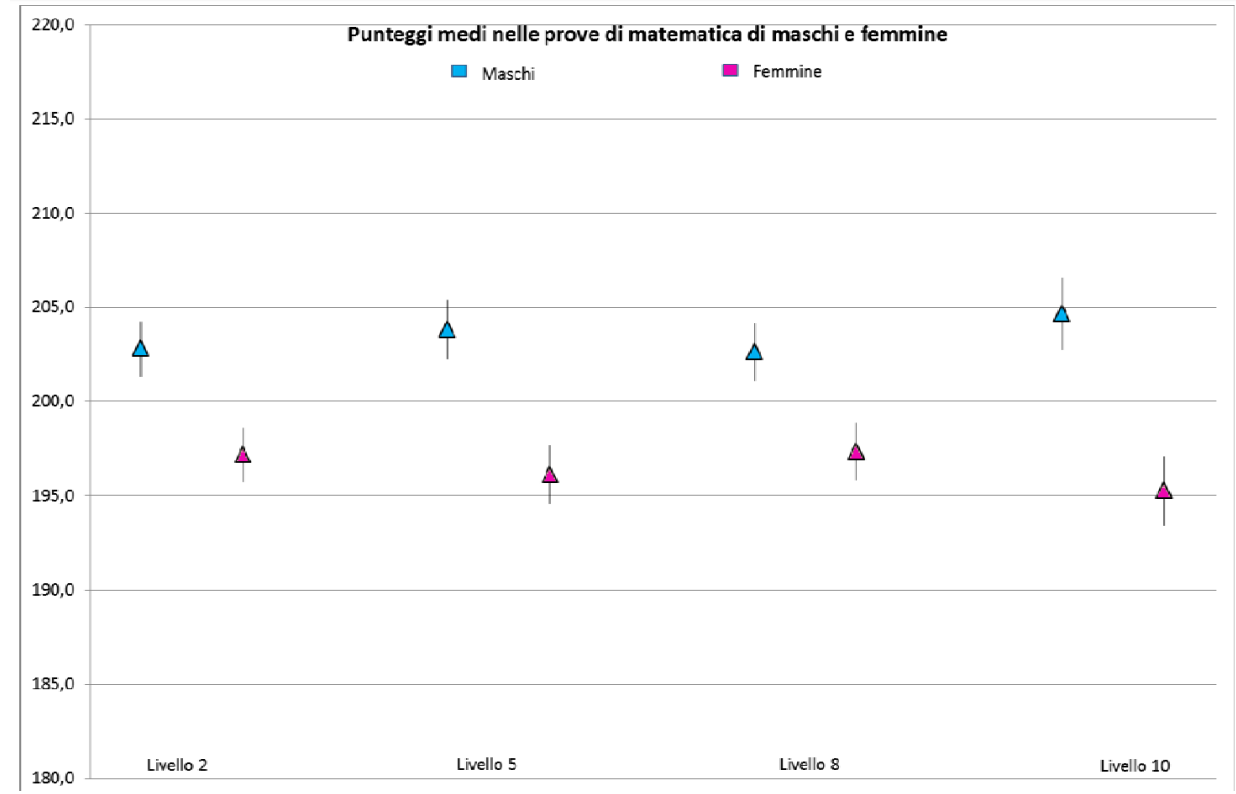

Per quanto riguarda i risultati del grado 10 , inoltre, si osserva che il divario tra i maschi e le femmine è presente in tutte le tipologie di scuola medio-superiore ma risulta più marcato se si passa dai professionali, ai tecnici e infine ai licei (INVALSI, 2017). 


\section{Differenze di genere in matematica: stato dell'arte}

Lo studio delle differenze di genere in matematica è un tema molto dibattuto negli ultimi decenni e sono numerose le ricerche che hanno cercato di indagare le caratteristiche e le cause del gender gap in matematica a favore dei maschi (Leder \& Forgasz, 2008; Forgasz, Becker, Lee \& Steinthorsdottir, 2010; Forgasz \& Rivera, 2012; Contini et al., 2017).

Nei prossimi paragrafi, sempre mantenendo un occhio di riguardo per la particolare situazione italiana, si cercherà di fornire una visione d'insieme delle caratteristiche del gender gap in matematica e dell'interpretazione delle sue cause per come emergono dalle principali ricerche sul fenomeno.

\subsection{Evoluzione del gender gap nei diversi livelli scolastici}

In base a quanto riportano diverse ricerche, le differenze di genere in matematica non sono già presenti in età prescolare ma emergono durante i primi anni di scuola. Al termine della scuola elementare il gap nelle performance di maschi e femmine è evidente e continua ad aumentare alle scuole medio-superiori (Fryer \& Levitt, 2010; Hyde \& Mertz, 2009; Hyde, Fennema \& Lamon, 1990; Spelke, 2005).

Fryer e Levitt (2010) hanno analizzato le performance in matematica di bambini e bambine seguendoli dalla scuola dell'infanzia fino al termine della scuola elementare (grado 5). In questo modo hanno evidenziato che il gap inizia nei primi anni di scuola e aumenta con il passare degli anni.

Ad oggi, dopo diversi anni dalla loro introduzione, le prove INVALSI somministrate in Italia possono essere utilizzate per studi simili a quello di Fryer e Levitt, sviluppato negli USA. In un recente studio basato sulle prove INVALSI, Contini et al. (2017), osservano l'evoluzione del gender gap in matematica anche nel contesto italiano e notano un complessivo ampliamento del divario a favore dei maschi dal grado 2 al grado 10; l'aumento del gap risulta però concentrato negli anni della scuola elementare e nel passaggio dal grado 8 al grado 10, rimanendo pressoché stabile negli anni della scuola media.

\subsection{Distribuzione del gender gap rispetto ai livelli di abilità}

Le prove standardizzate e I'uso di modelli statistici per l'analisi dei dati permettono anche di mettere in evidenza facilmente se un gap è particolarmente marcato per determinati livelli di abilità degli studenti.

Sempre dallo studio di Fryer e Levitt (2010), emerge che il divario tra maschi e femmine, a favore dei primi, risulta essere maggiore se si confrontano i risultati di studenti con alti livelli di abilità in matematica. Se alla scuola dell'infanzia le bambine occupano il $45 \%$ dei 5 percentili più alti, dopo cinque anni di scuola solo il $28 \%$ dei 5 percentili più alti è formato da femmine.

Anche dall'analisi dei dati PISA 2009, emerge che nella maggior parte dei Paesi partecipanti vi è un significativo gender gap in matematica a favore dei ragazzi ed è più marcato proprio tra gli studenti con alti livelli di abilità (González de San Román \& De La Rica, 2012). Questo dato viene confermato anche dai risultati dei test PISA 2012 e PISA 2015: pur osservando una notevole variabilità tra le nazioni coinvolte nell'indagine, le ragazze risultano sottorappresentate tra gli highest-achieving students 
(OECD, 2016a; OECD, 2012). Come è stato precedentemente osservato, nel 2015 il gap medio tra le performance di maschi e femmine nei Paesi OECD è di 8 punti ma il divario raddoppia, se si considera solamente il 10\% delle migliori performance maschili e il 10\% delle migliori performance femminili (OECD, 2016a).

Dalle rilevazioni PISA, I'Italia è uno dei Paesi in cui questo fenomeno risulta più evidente e nel 2015 solo I' $8 \%$ delle ragazze raggiunge il livello maggiore nella scala PISA contro il $13 \%$ dei ragazzi (OECD, 2016a). Contini et al. (2017) confermano questi risultati anche analizzando i dati INVALSI e, attraverso uno studio longitudinale delle prove dal grado scolastico 2 al grado 10, notano che al grado 2 il gap di genere non sussiste per i livelli di abilità più bassi ed è comunque poco marcato anche per i livelli di abilità medi e alti; con il passare degli anni questo gap compare anche per i livelli di abilità più bassi della distribuzione ma l'aumento del divario risulta molto più marcato per i livelli di abilità alti.

La ricerca sul tema del gender gap è molto controversa e in letteratura si trovano anche diversi studi che non evidenziano un gap statisticamente significativo. Come già sottolineato, i motivi possono essere legati alla non omogeneità geografica del fenomeno e quindi dagli stati coinvolti nelle rilevazioni, ma una seconda causa può anche essere la non omogeneità del gap rispetto al livello di abilità degli studenti. Un articolo che ha suscitato notevole scalpore in questo senso è stato quello pubblicato da Hyde, Lindberg, Linn, Ellis, e Williams nel 2008 sulla rivista Science. Hyde e colleghi, analizzando i dati di prove standardizzate degli Stati Uniti, concludono che «In contrasto con i risultati precedenti, questi dati non forniscono alcuna prova di una differenza di genere che favorisca i maschi e che emerga negli anni di scuola superiore» (Hyde et al., 2008, p. 494, traduzione dell'autore). Nello stesso anno, Leder e Forgasz (2008) mettono in luce le possibili cause che hanno portato Hyde e colleghi a risultati discordanti con la maggior parte delle ricerche nel settore. Tra le motivazioni riportate, la principale è quella per cui le differenze di genere si evidenziano per livelli alti di abilità. Leder e Forgasz spiegano che l'uso, da parte di Hyde e colleghi, di test formati soprattutto da item di livello cognitivo medio e basso, può essere la causa per cui non emerge gender gap.

Conoscere le peculiarità di questo fenomeno permette quindi anche una riflessione approfondita su quale sia uno strumento adatto per rilevarlo al meglio: la costruzione dei test dovrebbe sempre tenere conto anche di questi fattori.

\subsection{Interpretazione dei fattori che influiscono sul gender gap}

Per quanto riguarda I'individuazione dei fattori che sono alla base del gender gap, la letteratura è molto ampia e dibattuta: si possono trovare interpretazioni di natura biologica, sociale, culturale e psicologica che devono essere considerate congiuntamente per comprendere a pieno questo fenomeno. In particolare, è possibile distinguere tra fattori cosiddetti interni (o dipendenti dall'individuo) e fattori esterni (cioè dipendenti dal contesto socio-culturale e ambientale).

\section{Fattori interni}

Alcuni studi considerano tra i fattori interni anche differenze di tipo biologico e fisiologico (Baron-Cohen \& Wheelwright, 2004; Gallagher \& Kaufman, 2004) che permetterebbero ai maschi di sviluppare meglio alcune abilità legate all'apprendimento della matematica. D'altra parte la variabilità del fenomeno tra un Paese e I'altro (come restituita dalle rilevazioni internazionali) sembra mostrare i limiti di tale 
interpretazione, almeno come interpretazione unica (Contini et al., 2017; OECD, 2016a; Hill et al., 2010).

Le differenze relative alle principali abilità cognitive non sembrano essere tali da spiegare il gender gap in matematica. Molti studi, infatti, sostengono che non esistano differenze significative in termini di abilità cognitive generali (Ruffing, Wach, Spinath, Brünken \& Karbach, 2015; Halpern, Beninger \& Straight, 2011). Solo nel caso delle abilità visuo-spaziali, alcune ricerche riscontrano migliori risultati da parte degli studenti maschi (Lawton \& Hatcher, 2005). D'altra parte, tali abilità possono essere facilmente sviluppate attraverso un training mirato e di breve durata: il gap in questo settore potrebbe essere quindi facilmente colmato (Hill et al., 2010).

Tra i fattori strettamente collegati all'individuo che sono stati studiati in relazione al gender gap ci sono anche i fattori psico-sociali legati alle motivazioni, alle convinzioni degli studenti e alla fiducia nelle proprie capacità (Winkelmann, van den Heuvel-Panhuizen \& Robitzsch, 2008). Già nella scuola elementare, si osserva una minore fiducia nelle proprie abilità in matematica da parte delle femmine e, anche quando i risultati tra i due generi sono simili, sembra che le studentesse tendano a sottostimare le proprie capacità rispetto ai coetanei maschi (OECD, 2015; Fredericks \& Eccles, 2002; Herbert \& Stipek, 2005).

In questa direzione, I'OECD analizza principalmente tre costrutti legati alla matematica: math self-concept, math self-efficacy e math anxiety. ${ }^{5}$ I primi due costrutti rientrano all'interno di quelli che sono noti come self-beliefs e sono strettamente interconnessi pur riflettendo aspetti diversi riguardanti la sfera delle convinzioni, delle motivazioni e delle emozioni degli studenti in matematica. Infatti, la self-efficacy riguarda le sensazioni e le convinzioni degli studenti nel momento in cui devono risolvere uno specifico quesito (ad esempio risolvere un'equazione) mentre nel caso del math self-concept le domande rivolte agli studenti sono più generali e legate alla disciplina nel suo complesso, non a uno specifico task (Pajares \& Miller, 1994).

II ruolo della self-efficacy e del self-concept risulta essere fondamentale nell'apprendimento di qualsiasi disciplina e in particolare nel caso della matematica (OECD, 2015; Marsh \& O’Mara, 2008): uno studente con scarsa fiducia nei propri mezzi, infatti, sarà meno sicuro davanti a un compito e, nel caso in cui si trovi in difficoltà, sarà meno propenso a perseverare per raggiungere l'obiettivo.

I risultati riportati relativi a questi fattori sono tratti dall'indagine PISA 2012 in quanto, essendo la matematica il focus dell'indagine di quell'anno, anche i questionari di contesto erano mirati a indagare aspetti relativi all'apprendimento della matematica. Da tali questionari è possibile quindi avere anche informazioni relative ad aspetti legati alla sfera psicologica e metacognitiva dell'apprendimento della matematica e, in particolare, nel rapporto OECD PISA 2012 emerge che, anche nella scuola medio-superiore, permane una notevole differenza tra ragazzi e ragazze sia in termini di self-efficacy sia in termini di self-concept in matematica.

5. Le definizioni fornite dall'OECD degli indici utilizzati per analizzare questi costrutti sono le seguenti (OECD, 2013, traduzione dell'autore):

- Mathematics self-concept: indice basato sulle risposte degli studenti riguardanti la loro competenza percepita in matematica.

- Mathematics self-efficacy: indice basato sulle risposte degli studenti riguardanti la loro abilità percepita nel risolvere una varietà di problemi di matematica pura e applicata.

- Mathematics anxiety: indice basato sulle risposte degli studenti riguardo alle sensazioni di stress e bisogno di aiuto nell'affrontare la matematica. 
Figura 6

Self-concept in matematica in relazione al genere dati relativi all'indagine OECD PISA 2012. Le differenze statisticamente significative sono evidenziate con colori più scuri (in questo caso risultano tutte significative). Fonte: The $A B C$ of Gender Equality in Education: Aptitude, Behaviour, Confidence. (OECD, 2015).
Gender differences in mathematics self-concept

OECD average percentage of students who agreed or strongly agreed with the following statements:

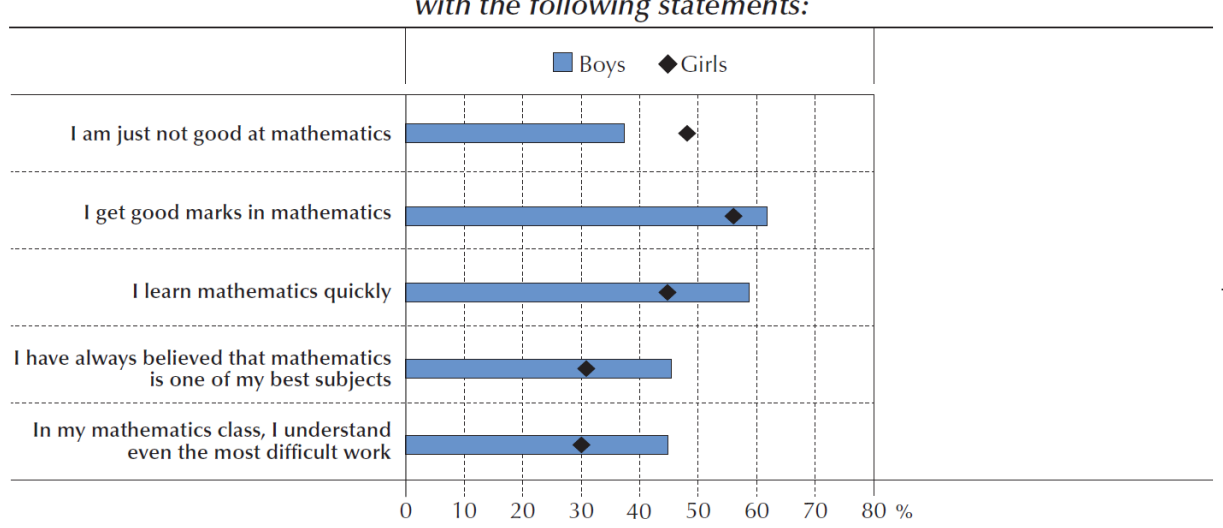

Gender differences in mathematics self-efficacy

$O E C D$ average percentage of students who reported that they can:

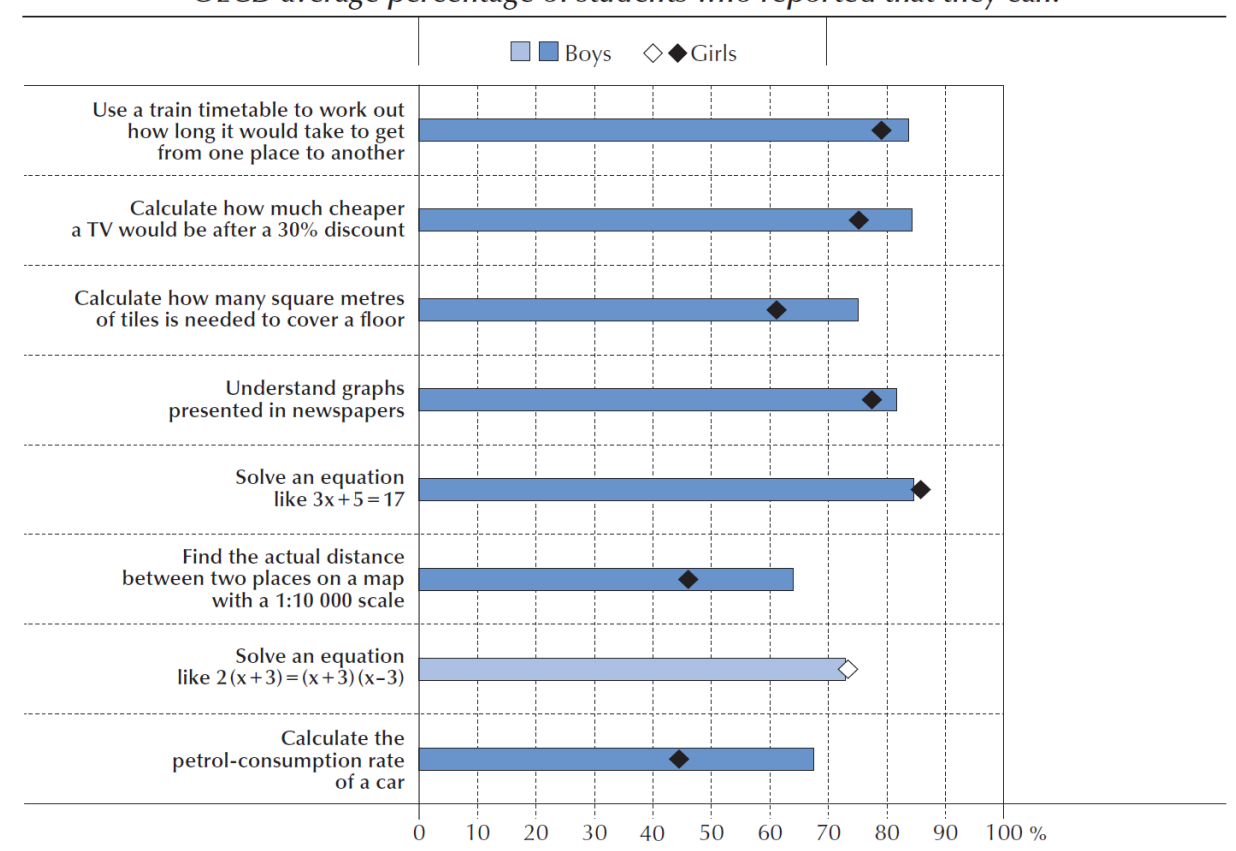

Come si osserva nei grafici sopra riportati (Figura 6 e Figura 7), relativi all'indagine PISA 2012, in generale le ragazze mostrano una minor fiducia nelle proprie capacità in matematica.

Le studentesse si considerano meno brave in matematica rispetto agli studenti maschi, pensano di non essere veloci nell'apprendimento della disciplina e dicono di trovarsi in difficoltà specialmente davanti agli argomenti più complessi (Figura 6). Sempre dallo stesso grafico, relativo al math self-concept, si evince che le risposte di maschi e femmine sono quantitativamente più vicine solo nel caso in cui si chiede di rispondere basandosi su un riferimento esterno, come i voti scolastici. Dalle indagini OECD si desume inoltre che la differenza tra maschi e femmine relativamente al math self-concept è presente anche a parità di abilità in matematica o a parità di risultati nella prova e questi risultati sono in accordo con letteratura precedente relativa a self beliefs e gender gap in matematica (OECD, 2015; Jacobs, Lanza, Osgood, Eccles \& Wigfield, 2002; Hill et al., 2010). 
Per quanto riguarda la math self-efficacy è interessante notare (Figura 7) che gli unici due task in cui la differenza non è a favore dei maschi risultano essere quelli in cui si richiede di risolvere un'equazione: si tratta in questo caso di esercizi risolvibili attraverso l'applicazione di procedure di routine, già svolte in classe che, a quanto pare, permettono alle ragazze di avere una maggiore fiducia nelle proprie capacità di risoluzione.

Un altro costrutto molto importante per analizzare i diversi risultati in matematica di maschi e femmine è l'ansia matematica (math anxiety), definita come «una reazione emotiva avversa alla matematica o alla prospettiva di fare matematica» (Hembree, 1990, traduzione dell'autore).

Numerosi studi hanno mostrato quanto l'essere ansiosi, spaventati e tesi nell'affrontare un compito di matematica porti gli studenti ad ottenere risultati inferiori rispetto alle proprie abilità (Hembree, 1990; Ma, 1999; Dowker, Sarkar \& Looi, 2016; Primi et al., 2014; OECD, 2015).

Dai risultati OECD relativi alla prova PISA del 2012 emerge una netta differenza nei livelli di ansia tra ragazzi e ragazze (Figura 8) confermata anche da numerosi altri studi sia a livello internazionale (Dowker et al., 2016; Hembree, 1990; Devine, Fawcett, Szúcs \& Dowker, 2012), sia a livello nazionale (Primi et al., 2014; Cargnelutti, Tomasetto \& Passolunghi, 2016).

Figura 8

Math anxiety in relazione

al genere, dati relativi

all'indagine OECD PISA

2012. Le differenze stati-

sticamente significative

sono evidenziate con colo-

ri più scuri (in questo caso

risultano tutte significati-

ve). Fonte: The $A B C$ of

Gender Equality in Educa-

tion: Aptitude, Behaviour

Confidence. (OECD, 2015)
Gender differences in mathematics anxiety

$O E C D$ average percentage of students who agreed or strongly agreed with the following statements:

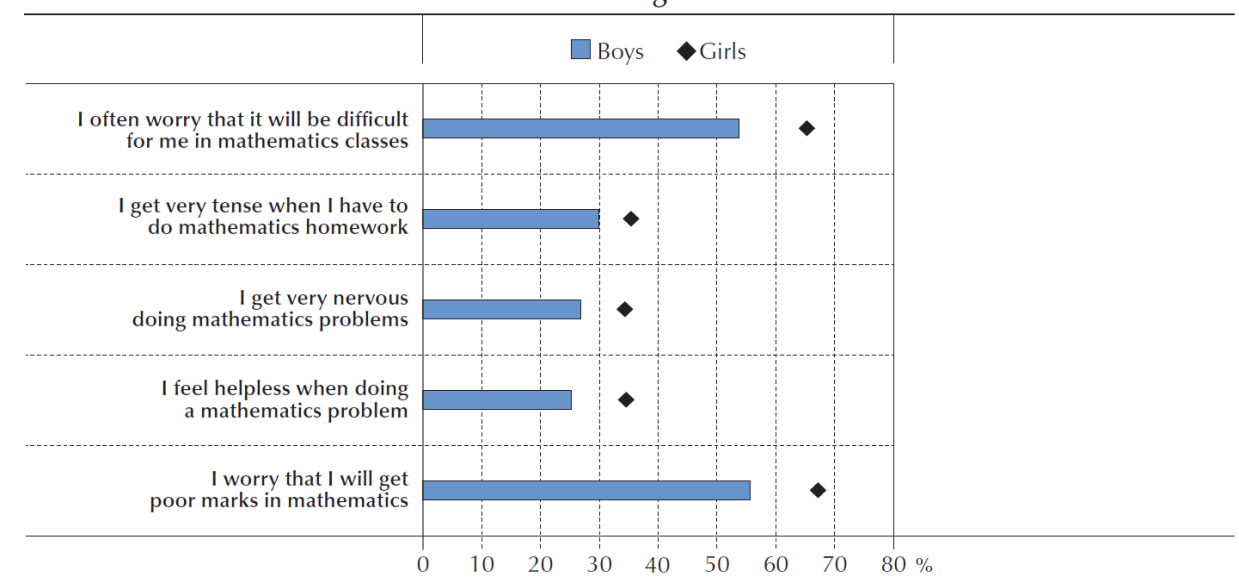

È interessante osservare che, se si considerano studenti e studentesse con pari livelli di math anxiety e math self-beliefs, il gap nei risultati dei test di matematica scompare (OECD, 2015).

Merita un discorso a parte l'analisi dei risultati in matematica in relazione al modello della self regulation, definita come l'abilità di controllare, dirigere e pianificare i propri pensieri, emozioni e comportamenti (Schunk \& Zimmerman, 1997). Dal punto di vista della disciplina, del rispetto delle regole, della partecipazione e dell'autocontrollo le ragazze risultano in generale migliori dei ragazzi (OECD, 2015; Matthews, Ponitz \& Morrison, 2009; Calkins, 2007; McClelland \& Cameron, 2011) e questo le favorisce anche nel rendimento scolastico nelle diverse discipline e tra queste la matematica. In un recente studio svolto in Germania (Weis, Heikamp \& Trommsdorff, 2013) viene evidenziato che, anche se non si riscontra una differenza di genere signi- 
ficativa considerando esclusivamente il rendimento in matematica, questo risultato può essere frutto anche di una migliore self-regulation da parte delle ragazze.

\section{Fattori esterni}

Per spiegare le differenze di genere in matematica, i fattori interni, di natura biologica e psicologica, devono essere accompagnati da altri fattori, questa volta esterni all'individuo e legati al contesto sociale e culturale in cui lo studente vive. In questa prospettiva, sono diverse le ricerche che hanno messo in relazione il gender gap in matematica con i principali indici di equità di genere utilizzati in campo economico e sociale. Queste ricerche hanno mostrato che nelle società in cui vi è una maggiore parità rispetto al genere, il gap tende a scomparire (Guiso, Monte, Sapienza \& Zingales, 2008; OECD, 2015; Cascella, 2017).

Uno studio particolarmente significativo in questo senso è quello di Guiso et al. (2008) basato sull'analisi della rilevazione OECD PISA del 2003. Gli autori mettono in relazione il gender gap in matematica e in italiano (grafico sopra in Figura 9) con uno dei principali indici utilizzati per evidenziare il gap di genere nella società: $\mathrm{GGI}$ World Economic Forum's Gender Gap Index (grafico sotto in Figura 9). Questo indice riflette, per ogni nazione, la parità di genere in base alle condizioni economiche, politiche, educative e di salute.

Figura 9

Analisi dei risultati del test PISA 2003 di comprensione del testo e di matematica in relazione al livello di parità dei generi nella società. Fonte: Guiso et al., 2008.

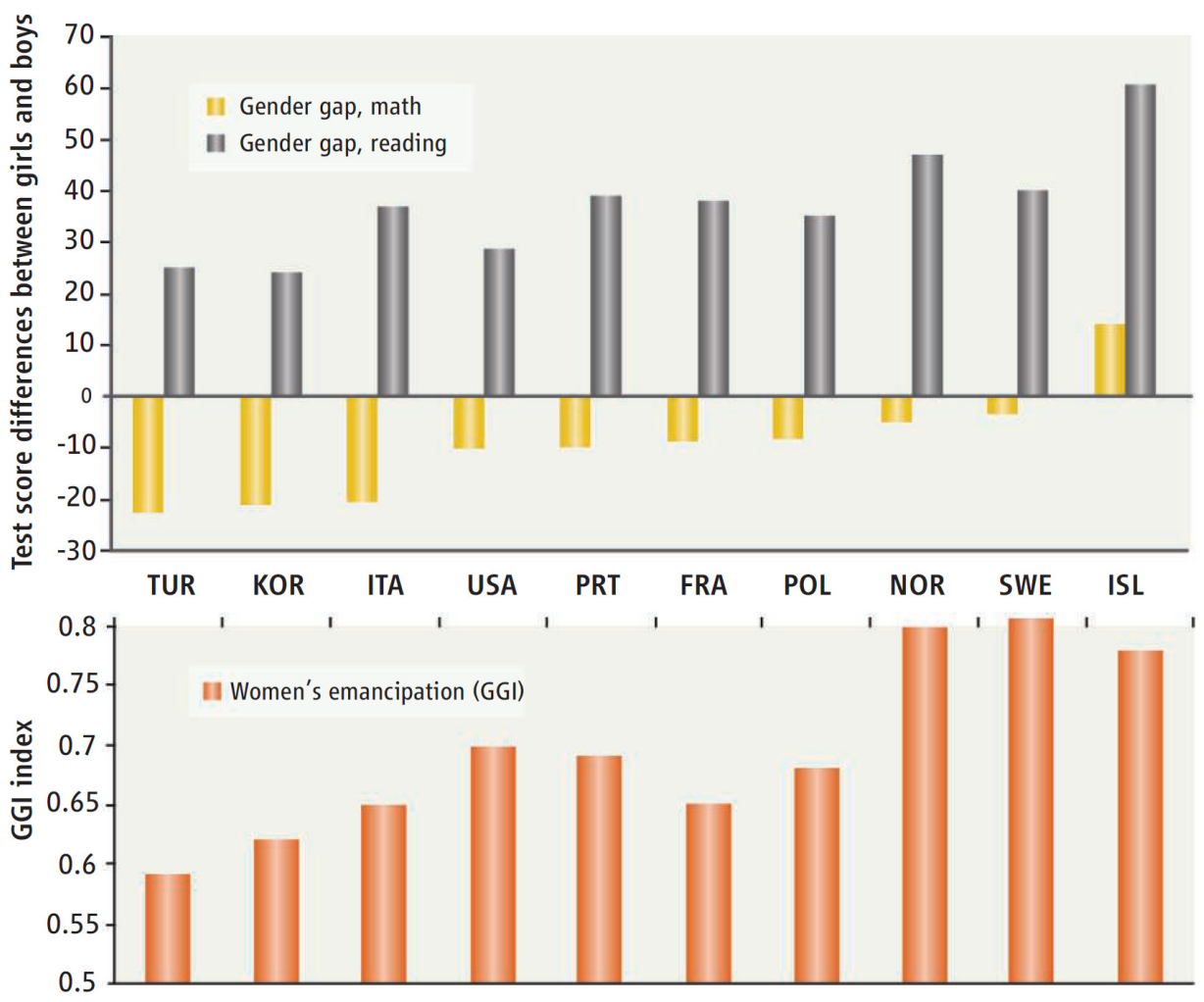

Nei grafici sopra si può confrontare, per ognuna delle nazioni prese in esame, il valore del Gender Gap Index (GGI) con i risultati del test PISA 2003 in termini di gender gap nella prova di matematica e lettura. Guiso et al. (2008) osservano che, nei Paesi in cui il ruolo della donna nella società ha raggiunto alti livelli di emancipazione (GGI maggiore), il gender gap in matematica tende ad annullarsi e il gap nella 
comprensione del testo a favore delle ragazze incrementa. Questi risultati vengono confermati, nello stesso articolo, anche attraverso l'uso di altri indici statistici basati anche su fattori di natura culturale.

Dal 2006 al 2017, il Global Gender Gap Report mostra come I'Italia si trovi in una situazione piuttosto critica in fatto di parità di genere: nel 2017 I'Italia è risultata 82-esima su 144 Paesi, risultato di gran lunga peggiore rispetto alla maggior parte dei Paesi europei.

Le analisi di Guiso e colleghi sono state replicate anche da Fryer e Levitt (2010) e i risultati relativi all'indagine OECD PISA sono stati confermati. Le stesse analisi, però, sono state ripetute anche utilizzando i risultati dell'indagine IEA TIMSS 2003 e, in questo caso, non si riscontra la stessa correlazione tra la riduzione del gender gap in matematica e l'equità di genere nella società. Fryer e Levitt spiegano che queste differenze sono legate al fatto che sono diverse le nazioni che partecipano alle due indagini: restringendo i risultati del TIMSS alle nazioni che partecipano anche all'indagine PISA, la correlazione tra GGI e la diminuzione del divario in matematica emerge nuovamente. Le nazioni che partecipano esclusivamente alle prove TIMSS sono principalmente Paesi del Medio Oriente, in cui il ruolo della donna risulta essere molto svantaggiato nella società ma, inaspettatamente, i risultati in matematica non sembrano risentirne (Fryer \& Levitt, 2010), probabilmente questo può essere anche dovuto a problemi di rappresentatività del campione, come già spiegato nei paragrafi precedenti.

Questi studi mostrano quanto sia fondamentale il ruolo della cultura e della società nell'educazione degli studenti e quanto raggiungere una reale parità sociale dei generi influisca positivamente sulle possibilità delle generazioni future. Alcune ricerche si sono occupate delle conseguenze economiche e sociali causate dalle disparità di genere nell' istruzione e, ad esempio, hanno riscontrato una minore crescita economica nei Paesi in cui le disuguaglianze sono maggiori (Klasen, 2002). Inoltre, politiche educative che mirano al raggiungimento di pari opportunità dei generi nel campo dell' istruzione vanno a vantaggio delle generazioni future in quanto si è visto che, se le donne e le madri raggiungono ruoli di maggiore emancipazione nella società, questo influisce positivamente sull'istruzione e sulla salute dei figli (Schultz, 2002; Doepke, Tertilt \& Voena, 2011; Farré \& Vella, 2013).

Sono molteplici gli studi che sottolineano l'importanza del ruolo che la madre assume all'interno della famiglia e della società e come questo influenzi le performance in matematica dei figli e in particolare delle figlie femmine (Fryer \& Levitt, 2010; Jacobs \& Eccles, 1992; González de San Román \& De La Rica, 2012).

L'apprendimento della matematica e la disparità nei risultati in questa disciplina sono influenzati dal contesto, dalle caratteristiche socio-economiche e culturali dei singoli Paesi e dal ruolo della donna all'interno della società. Esistono anche studi basati su indagini più recenti che hanno confermato queste ipotesi e indagato approfonditamente il rapporto tra emancipazione della donna e gender gap in matematica. Ad esempio, nella ricerca di González de San Román e De La Rica (2012), attraverso l'analisi dei risultati PISA 2009, viene confermata la forte influenza delle norme sociali e culturali del Paese sulle differenze di genere riscontrate nei test.

Si considerano rilevanti, nel determinare le differenze di genere osservate, anche fattori legati maggiormente alle convinzioni e alla sfera psico-sociale di natura esterna all'individuo. Giocano infatti un ruolo fondamentale le convinzioni di insegnanti e genitori riguardo alle diverse abilità di maschi e femmine in matematica e gli stereotipi che vedono i ragazzi come più portati per le materie scientifiche men- 
tre le ragazze favorite nelle materie letterarie.

Si è riscontrato che tutto ciò incide notevolmente sulla percezione degli studenti riguardo alle proprie possibilità (convinzioni su di sé) e influisce quindi sulle loro reali performance (Jacobs \& Bleeker, 2004; Riegle-Crumb, 2005; Fryer \& Levitt, 2010). Gli stereotipi legati al genere che affondano le loro radici nella cultura del nostro Paese e di molti altri, portano avanti l'idea che i maschi siano naturalmente più portati per le materie scientifiche e influenzano anche il modo in cui i genitori si rivolgono ai figli fin dai primi anni di vita (Jacobs \& Bleeker, 2004; Tomasetto, 2013; Tomasetto, Mirisola, Galdi \& Cadinu, 2015). Questi hanno comportamenti e aspettative diversi nei confronti di un figlio maschio o di una figlia femmina e una percezione diversa relativamente alle loro abilità e ai successi in matematica (Tiedemann, 2000; Tomasetto, 2013). Tutto ciò si riflette sulla percezione che lo studente ha delle proprie abilità, andando quindi a sfavorire le ragazze nelle materie scientifiche e in particolare in matematica (Jacobs \& Eccles, 1992; Spinath \& Spinath, 2005; Tomasetto, 2013). Risulta molto interessante anche il fatto che siano diverse, in base al sesso del figlio, le motivazioni che i genitori adducono per giustificare un successo o un insuccesso in matematica: i successi dei figli maschi vengono spesso associati ad una predisposizione naturale per la disciplina mentre per le femmine un successo è ritenuto più spesso frutto di impegno e costanza; per quanto riguarda gli insuccessi invece $\mathrm{i}$ genitori tendono a motivarli con una mancanza di impegno dei figli maschi e a una scarsa abilità delle figlie femmine (Eccles, Jacobs \& Harold, 1990; Yee \& Eccles, 1988; Tomasetto, 2013).

Gli stereotipi non riguardano però solamente i genitori; anche gli insegnanti infatti mostrano di essere influenzati dalle medesime convinzioni e tendono a considerare i maschi con maggiori abilità in matematica rispetto alle ragazze (Helwig, Anderson \& Tindal, 2001; Li, 1999).

L'impatto che le convinzioni di insegnanti e genitori hanno, relativamente alle abilità degli studenti, è rilevante e il fatto che le studentesse vengano ritenute meno dotate in matematica rispetto ai coetanei maschi fa sì che esse stesse abbiano meno fiducia nelle proprie abilità e ottengano quindi risultati effettivamente minori (Lindberg et al., 2010).

Infine, anche fattori strettamente collegati al contesto scolastico e alle pratiche didattiche possono fornire una possibile chiave di lettura delle differenze di genere in matematica. In un articolo del 1992, Leder evidenzia tra le possibili cause delle differenze di genere in matematica anche variabili legate ai curricula specifici della disciplina: gli argomenti trattati, la tipologia di quesiti, i metodi di valutazione e insegnamento hanno un ruolo fondamentale nell'emergere delle differenze di genere in matematica. Studi più recenti hanno confermato questa ipotesi e hanno mostrato che, oltre a variabili connesse ai curricula, anche i metodi di insegnamento e di valutazione, le pratiche didattiche e le norme socio-matematiche che si instaurano nella classe hanno una notevole influenza sul gender gap in matematica (Leder \& Forgasz, 2008; OECD, 2016; Giberti, Zivelonghi \& Bolondi, 2016; Bolondi, Cascella \& Giberti, 2017; Bolondi, Branchetti \& Giberti, 2018; Ferretti, Giberti \& Lemmo, 2018; Bolondi, Ferretti \& Giberti, 2018).

\section{Learning abilities e learning strategies}

Come detto, tra i fattori che influenzano il gender gap in matematica ci sono anche variabili legate ai contenuti specifici della matematica. Risulta quindi necessario analizzare il gender gap non solo da un punto di vista dell'intera disciplina, ma declinan- 
do l'abilità matematica nelle sue molteplici componenti e considerando i diversi processi cognitivi che gli studenti devono attivare nella risoluzione di un task matematico. Le studentesse mostrano una maggiore difficoltà rispetto ai compagni maschi nell'approccio ad attività di problem solving, mentre mostrano capacità equivalenti o leggermente superiori in compiti che richiedono principalmente abilità di calcolo (Hyde et al., 1990; Byrnes \& Takahira, 1993). Altri studi hanno dimostrato come maschi e femmine abbiano un modo diverso di affrontare attività di problem solving e adottino quindi strategie differenti: le ragazze tendono maggiormente a ripetere procedure di routine, algoritmi già utilizzati e conosciuti, strategie convenzionali, mentre i ragazzi, mostrando meno paura di sbagliare, tendono ad applicare anche nuovi metodi e approcci non convenzionali (Bell \& Norwood, 2007; Gallagher, De Lisi, Holst, McGillicuddy-De Lisi, Morely \& Cahalan, 2000; Gould, 1996; Fennema, Carpenter, Jacobs, Franke \& Levi, 1998).

Queste evidenze, oggi confermate da recenti studi anche in ambito psicologico, erano già state presentate nel 1998 da Fennema e Carpenter, attraverso uno studio longitudinale riguardante i primi anni della scuola elementare. I ricercatori non riscontrano particolari differenze di genere se non nel caso di una tipologia di problemi (extension problems) ed esclusivamente a grado 3, al contempo però

«in tutti i gradi scolastici sono state evidenziate differenze significative nelle strategie adottate in attività di problem solving. Le ragazze tendono a usare strategie risolutive concrete come la modellizzazione e il conteggio, mentre i ragazzi tendono a utilizzare strategie risolutive più astratte che riflettono una comprensione a livello concettuale».

(Fennema \& Carpenter, 1998, p. 4, traduzione dell'autore)

Anche in questo caso, le differenti strategie di problem solving non sembrano strettamente legate a caratteristiche biologiche diverse tra maschi e femmine ma piuttosto a fattori di tipo socio-culturale e legati alla sfera delle convinzioni. Tra questi ritroviamo sicuramente la minore fiducia nelle proprie abilità da parte delle ragazze, stereotipi largamente diffusi per cui, ad esempio, "le brave ragazze seguono le regole" ("good girls follow the rules" - Langer, 1997) e fattori legati alla didattica, al sistema scolastico e alle pratiche d'aula (Boaler, 1997).

In questa direzione risulta particolarmente interessante il confronto con altre ricerche legate alle strategie di apprendimento applicate dagli studenti nello studio delle diverse materie. Non si riscontrano differenze in termini di abilità cognitive (Halpern et al., 2011), si hanno però differenze di genere significative per quanto riguarda le strategie d'apprendimento (Kesici, Sahin \& Akturk, 2009; Marrs \& Sigler, 2012; Virtanen \& Nevgi, 2010).

La letteratura mostra quanto abilità cognitive e strategie d'apprendimento abbiano una forte influenza sulle performance degli studenti; questo è vero anche quando si vuole predire il successo accademico (Rohde \& Thompson, 2007; Richardson, Abraham \& Bond, 2012). Un recente articolo Ruffing et al. (2015) trattano proprio le differenze di genere e le strategie di apprendimento in funzione del loro impatto sulle performance accademiche. Da questo articolo emerge che non vi sono particolari differenze in termini di abilità cognitive, ma che maschi e femmine mostrano un diverso approccio rispetto alle strategie di apprendimento prese in considerazione. In particolare, vengono considerate le seguenti strategie d'apprendimento: 
- Effort

- Attention

- Organization

- Relationship

- Rehearsal

- Critical evaluation

- Time management

- Learning environment

- Learning with fellow students

- Literature

- Meta-cognition

Fra queste ritroviamo solo due strategie che risultano essere maggiormente utilizzate dai maschi rispetto alle femmine: Relationship e Critical Evaluation. Le ragazze, invece, mostrano un maggiore uso di molte delle rimanenti strategie: Effort, Organization, Rehearsal, Time Management e Meta-Cognition (Ruffing et al., 2015).

Le differenze nelle strategie di apprendimento possono chiaramente essere alla base di un apprendimento con caratteristiche molto diverse per maschi e femmine. Questi risultati potrebbero essere una possibile spiegazione di alcuni fenomeni osservati nell'apprendimento della matematica: le ragazze, concentrandosi soprattutto sull'impegno, sull'organizzazione dello studio e sulla ripetizione dei concetti, potrebbero essere maggiormente legate alle procedure già viste in classe o durante lo studio a casa ed essere meno pronte a rispondere a stimoli nuovi e che richiedono un apprendimento più basato sulla comprensione profonda dei concetti al fine di una rielaborazione.

\section{Conclusioni}

I risultati delle prove standardizzate risultano essere un ottimo strumento per lo studio del gender gap in matematica e permettono di evidenziare le principali caratteristiche di questo fenomeno. In particolare, dall'analisi delle prove PISA e TIMSS si evince che non in tutte le nazioni è presente un gender gap statisticamente significativo a favore dei maschi e questo porta a escludere, tra le principali cause delle differenze di genere, quelle di natura biologica, supportando invece l'ipotesi che la natura di questo divario sia piuttosto da imputare a fattori di natura sociale e culturale. Le ricerche mostrano che non è possibile individuare un'unica causa del gender gap in matematica, ma che piuttosto vi sono molteplici e differenti fattori da considerare. Quelli sociali e culturali sono particolarmente importanti come, ad esempio, il livello di emancipazione della donna nella società. Gli stereotipi, legati alla storia e alla cultura della società e dello stesso nucleo familiare, sono un altro fattore importante perché hanno un impatto sull'approccio alla disciplina e sui fattori psicologici e metacognitivi. Questi fattori possono portare a un diverso approccio alla disciplina e influenzare anche il modo di affrontare una specifica consegna matematica.

Le differenze evidenziate in psicologia tra maschi e femmine, come una maggiore math anxiety e una minore fiducia nelle proprie capacità per le ragazze, accompagnate però da un maggiore controllo e disciplina, possono avere una notevole in- 
fluenza sull'atteggiamento degli studenti in classe e sul rapporto che si instaura con I'insegnante e nei confronti della matematica.

Si riscontrano inoltre significative differenze in termini di strategie di apprendimento per maschi e femmine, una maggiore difficoltà da parte delle studentesse nell'affrontare attività di problem solving dovute anche a una maggiore paura di sbagliare e una maggiore reticenza nell'adottare nuove strategie risolutive a favore di algoritmi conosciuti e procedure di routine. Queste evidenze risultano particolarmente importanti per la ricerca in didattica e per gli insegnanti stessi che possono indirizzare l'azione didattica tenendo in considerazione queste differenze al fine di colmare questo divario.

Risulta infatti fondamentale affrontare questo fenomeno tenendo conto non solo delle cause di natura sociale e culturale a livello generale, ma anche di fattori micro-sociali, legati alle abitudini del contesto classe, al rapporto con l'insegnante e alle pratiche didattiche.

L'incidenza di fattori micro-sociali, legati alla didattica e alle pratiche d'aula, è supportata anche dal fatto che, come osservato nei paragrafi precedenti, le differenze di genere in matematica emergono durante i primi anni di scuola e aumentano con il passare degli anni ed è proprio a questo livello che l'azione del singolo insegnante può avere la massima efficacia.

Le prove PISA e TIMSS mostrano come in Italia il gap tra le performance dei maschi e delle femmine in matematica sia uno dei più marcati. Le prove nazionali INVALSI confermano questo divario che si presenta in tutti i livelli scolastici. In Italia, questo fenomeno è accompagnato da un peggioramento anche più generale del Gender Gap Index, che esprime la parità di genere prendendo in considerazione il divario tra uomini e donne in quattro ambiti: lavoro, istruzione, salute e rappresentanza politica. Risulta quindi necessaria una riflessione su due piani differenti: il primo politico, per capire come mai l'Italia risulta ancora in una situazione tanto critica in termini di parità di genere, I'altro didattico, per indagare più approfonditamente come queste disparità vadano a incidere sul rendimento in matematica.

La consapevolezza della presenza di questo fenomeno, particolarmente marcato in Italia, può portare, gli insegnanti e gli educatori in generale, a riflettere sulle proprie convinzioni e sull'approccio alla disciplina. La collaborazione tra gli insegnanti e il mondo della ricerca in didattica può portare a individuare quali siano le difficoltà che ostacolano le ragazze in matematica e se queste siano legate a particolari contenuti o ad abilità trasversali. In questo modo sarà possibile elaborare una didattica mirata a superare tali difficoltà per chiudere il gap in matematica tra maschi e femmine.

\section{Bibliografia}

Baron-Cohen, S., \& Wheelwright, S. (2004). The empathy quotient: an investigation of adults with Asperger syndrome or high functioning autism, and normal sex differences. Journal of autism and developmental disorders, 34(2), 163-175.

Bell, K. N., \& Norwood, K. (2007). Gender equity intersects with mathematics and technology: Problem-solving education for changing times. In D. Sadker \& E. S. Silber (Eds.), Gender in the classroom (pp. 225-258). Mahweh: Lawrence Erlbaum Associates.

Boaler, J. (1997). Reclaiming school mathematics: The girls fight back. Gender and Education, 9(3), 285-305. 
Bolondi, G., Branchetti, L., \& Giberti, C. (2018). A quantitative methodology for analyzing the impact of the formulation of a mathematical item on students learning assessment. Studies in Educational Evaluation, 58, 37-50.

Bolondi, G., Cascella, C., \& Giberti, C. (2017). Highlights on gender gap from Italian standardized assessment in mathematics. In J. Novotnà \& H. Moravà (Eds.), Diversity in Mathematics Education. Proceedings of the International Symposium Elementary Maths Teaching, SEMT 17. Prague: Universita Karlova Press.

Bolondi, G., Ferretti, F., \& Giberti, C. (2018). Didactic Contract as a key to Interpreting Gender Diffrences in Maths. Journal of Educational, Cultural and Psychological Studies (ECPS Journal), 18, 415-435.

Byrnes, J. P. (2005). Gender Differences in Math: Cognitive Processes in an Expanded Framework. In A. M. Gallagher \& J. C. Kaufman (Eds.), Gender differences in mathematics: An integrative psychological approach (pp. 73-98). New York, US: Cambridge University Press.

Byrnes, J. P., \& Takahira, S. (1993). Explaining gender differences on SAT-math items. Developmental Psychology, 29(5), 805.

Calkins, S. D. (2007). The emergence of self-regulation: Biological and behavioral control mechanisms supporting toddler competencies. In C. A. Brownell \& C. B. Kopp (Eds.), Socioemotional development in the toddler years: Transitions and transformations (pp. 261284). New York, US: Guilford Press.

Cargnelutti, E., Tomasetto, C., \& Passolunghi, M. C. (2016). How is anxiety related to math performance in young students? A longitudinal study of Grade 2 to Grade 3 children. COGNITION \& EMOTION, 31, 755-764.

Cascella, C., (2017). Exploring the relationship between social roles in daily life and achievement gap between boys and girls in maths: empirical evidences from Italian primary school. In 11th annual International Technology, Education and Development Conference Proceedings (pp. 9832-9841). Valencia: IATED Publisher. DOI: 10.21125/inted.2017.2339

Contini, D., Di Tommaso, M. L., \& Mendolia, S. (2017). The gender gap in mathematics achievement: Evidence from Italian data. Economics of Education Review, 58, 32-42.

Devine, A., Fawcett, K., Szűcs, D., \& Dowker, A. (2012). Gender differences in mathematics anxiety and the relation to mathematics performance while controlling for test anxiety. Behavioral and brain functions, 8(1), 33.

Doepke, M., Tertilt, M., \& Voena, A. (2011). The economics and politics of women's rights. Annual Review of Economics, 4, 339-372.

Dowker, A., Sarkar, A., \& Looi, C. Y. (2016). Mathematics anxiety: what have we learned in 60 years?. Frontiers in psychology, 7, 508.

Eccles, J. S., Jacobs, J. E., \& Harold, R. D. (1990). Gender role stereotypes, expectancy effects, and parents' socialization of gender differences. Journal of Social Issues, 46(2), 183-201.

Farré, L., \& Vella, F. (2013). The intergenerational transmission of gender role attitudes and its implications for female labour force participation. Economica, 80(318), 219-247.

Fennema, E., \& Carpenter, T. P. (1998). New Perspectives on Gender Differences in Mathematics: An Introduction. Educational Researcher, 27(5), 4-5.

Fennema, E., Carpenter, T. P., Jacobs, V. R., Franke, M. L., \& Levi, L. W. (1998). New perspectives on gender differences in mathematics: A reprise. Educational Researcher, 27(5), 19-21. 
Ferretti, F., Giberti, C., \& Lemmo, A. (2018). The Didactic Contract to Interpret Some Statistical Evidence in Mathematics Standardized Assessment Tests. EURASIA Journal of Mathematics, Science and Technology Education, 14 (7), 2895-2906. Disponibile in https://doi. org/10.29333/ejmste/90988 (consultato il 05.09.2018).

Forgasz, H. J., Becker, J. R., Lee, K., \& Steinthorsdottir, O. (Eds.). (2010). International perspectives on gender and mathematics education. Charlotte, NC: Information Age Publish

Forgasz, H. J., \& Rivera, F. (2012). Towards Equity in Mathematics Education. Springer.

Fredericks, J. A., \& Eccles, J. S. (2002). Children's Competence and Value Beliefs Childhood Adolescence: Growth Trajectories in Two Male-Sex-Typed Domains. Developmental Psychology, 38 (2), 519-533. Disponibile in https://doi.org/10.1037/0012-1649.38.4.519 (consultato il 05.09.2018).

Fryer, R. G., \& Levitt, S. D. (2010). An empirical analysis of the gender gap in mathematics. American Economic Journal: Applied Economics, 2(2), 210-240.

Gallagher, A. M., De Lisi, R., Holst, P. C., McGillicuddy-De Lisi, A. V., Morely, M., \& Cahalan, C. (2000). Gender differences in advanced mathematical problem solving. Journal of experimental child psychology, 75(3), 165-190.

Gallagher, A. M., \& Kaufman, J. C. (Eds.). (2004). Gender differences in mathematics: An integrative psychological approach. New York, US: Cambridge University Press.

Giberti, C., Zivelonghi, A., \& Bolondi, G. (2016). Gender differences and didactic contract: analysis of two INVALSI tasks on powers properties. In C. Csikos, A. Rausch \& J. Szitanyi (Eds.), Proceedings of the 40th Conference of the International Group for the Psychology of Mathematics Education (p. 275). Szeged: IGPME.

González de San Román, A., \& De La Rica, S. (2012). Gender gaps in PISA test scores: The impact of social norms and the mother's transmission of role attitudes. Estudios de Economía Aplicada, 34(1).

Gould, S. L. (1996). Strategies used by secondary school students in learning new concepts which require spatial visualization. [Unpublished doctoral dissertation], Teachers College, Columbia University, New York.

Guiso, L., Monte, F., Sapienza, P., \& Zingales, L. (2008). Culture, gender, and math. Science-New York then Washington, 320 (5880), 1164.

Halpern, D. F., Beninger, A. S., \& Straight, C. A. (2011). Sex differences in intelligence. The Cambridge handbook of intelligence, 253-272.

Helwig, R., Anderson, L., \& Tindal, G. (2001). Influence of elementary student gender on teachers' perceptions of mathematics achievement. The Journal of Educational Research, 95(2), 93-102.

Hembree, R. (1990). The nature, effects, and relief of mathematics anxiety. Journal for research in mathematics education, 21(1), 33-46.

Herbert, J., \& Stipek, D. (2005). The emergence of gender differences in children's perceptions of their academic competence. Journal of Applied Developmental Psychology, 26(3), 276295.

Hill, C., Corbett, C., \& St. Rose, , A. (2010). Why So Few? Women in Science, Technology, Engineering, and Mathematics. Washington: American Association of University Women. Disponibile in https://www.aauw.org/aauw check/pdf download/show pdf.php?file=why-sofew-research (consultato il 12.09.2018). 
Hyde, J. S., Fennema, E., \& Lamon, S. J. (1990). Gender differences in mathematics performance: a meta-analysis. Psychological bulletin, 107(2), 139.

Hyde, J. S., Lindberg, S. M., Linn, M. C., Ellis, A. B., \& Williams, C. C. (2008). Gender similarities characterize math performance. Science, 321(5888), 494-495.

Hyde, J. S., \& Mertz, J. E. (2009). Gender, culture, and mathematics performance. Proceedings of the National Academy of Sciences, 106(22), 8801-8807.

INVALSI. (2016). Rilevazione nazionale degli apprendimenti 2015-2016. Rapporto risultati. Frascati, IT: INVALSI. Disponibile in http://www.invalsi.it/invalsi/doc evidenza/2016/07 Rapporto Prove INVALSI 2016.pdf (consultato il 17.07.2018).

INVALSI. (2017). Rilevazione nazionale degli apprendimenti 2016-2017. Rapporto risultati. Frascati, IT: INVALSI. Disponibile in https://invalsi-areaprove.cineca.it/docs/file/Rapporto Prove INVALSI 2017.pdf (consultato il 17.07.2018).

Jacobs, J. E., \& Bleeker, M. M. (2004). Girls' and boys' developing interests in math and science: Do parents matter? New directions for child and adolescent development, 2004(106), 5-21.

Jacobs, J. E., \& Eccles, J. S. (1992). The impact of mothers' gender-role stereotypic beliefs on mothers' and children's ability perceptions. Journal of personality and social psychology, 63(6), 932-944.

Jacobs, J. E., Lanza, S., Osgood, D. W., Eccles, J. S., \& Wigfield, A. (2002). Changes in children's self-competence and values: Gender and domain differences across grades one through twelve. Child development, 73(2), 509-527.

Kesici, S., Sahin, I., \& Akturk, A. O. (2009). Analysis of cognitive learning strategies and computer attitudes, according to college students' gender and locus of control. Computers in Human Behavior, 25(2), 529-534.

Klasen, S. (2002). Low schooling for girls, slower growth for all? Cross-country evidence on the effect of gender inequality in education on economic development. The World Bank Economic Review, 16(3), 345-373.

Langer, E. J. (1997). The power of mindful learning. Reading, MA: Addison Wesley.

Lawton, C. A., \& Hatcher, D. W. (2005). Gender differences in integration of images in visuospatial memory. Sex roles, 53(9-10), 717-725.

Leder, G. C. (1992). Mathematics and gender: Changing perspectives. In D. A. Grouws (Ed.), Handbook of research on mathematics teaching and learning (pp. 597-622). New York: Macmillan.

Leder, G., \& Forgasz, H. (2008). Mathematics education: new perspectives on gender. ZDM The International Journal on Mathematics Education, 40(4), 513-518.

Li, Q. (1999). Teachers' beliefs and gender differences in mathematics: A review. Educational Research, 41(1), 63-76.

Lindberg, S. M., Hyde, J. S., Petersen, J. L., \& Linn, M. C. (2010). New trends in gender and mathematics performance: a meta-analysis. Psychological bulletin, 136(6), 1123.

Ma, X. (1999). A meta-analysis of the relationship between anxiety toward mathematics and achievement in mathematics. Journal for research in mathematics education, 30(5), 520-540. 
Mackinnon, N. (1990). Sophie Germain: or was Gauss a feminist? Math Gaz, 74(470), 346351.

Maffia, A., \& Giberti, C. (2016). Didattica della matematica e PISA: strade percorse e nuovi sentieri da battere. In L. Palmerio (A cura di), PISA 2012. Contributi di approfondimento (pp. 190-200). Roma: Franco Angeli.

Marrs, H., \& Sigler, E. A. (2012). Male academic performance in college: The possible role of study strategies. Psychology of Men \& Masculinity, 13(2), 227.

Marsh, H. W., \& O’Mara, A. J. (2008). Reciprocal effects between academic self-concept, self-esteem, achievement, and attainment over seven adolescent years: Unidimensional and multidimensional perspectives of self-concept. Personality and Social Psychology Bulletin, 34, 542-552.

Matthews, J. S., Ponitz, C. C., \& Morrison, F. J. (2009). Early gender differences in self-regulation and academic achievement. Journal of educational psychology, 101(3), 689.

McClelland, M. M., \& Cameron, C. E. (2011). Self-regulation and academic achievement in elementary school children. New Directions for Child and Adolescent Development, 2011(133), 29-44.

Mullis, I. V. S., Martin, M. O., Foy, P., \& Hooper, M. (2016). TIMSS 2015 International Results in Mathematics. Boston: TIMSS \& PIRLS International Study Center.

OECD. (2012). Results in Focus: What 15-year-olds know and what they can do with what they know. Paris, FR: OECD Publishing.

OECD. (2013). PISA 2012 Results: Ready to Learn: Students' Engagement, Drive and Self-Beliefs. Paris, FR: OECD Publishing.

OECD. (2015). The ABC of Gender Equality in Education: Aptitude, Behaviour, Confidence. Paris, FR: OECD Publishing.

OECD. (2016a). PISA 2015 Results (Volume I): Excellence and Equity in Education. Paris, FR: OECD Publishing.

OECD. (2016b). ITALY - Country Note - Results from PISA 2015. Paris, FR: OECD Publishing.

OECD. (2016c). PISA 2015 Assessment and Analytical Framework: Science, Reading, Mathematic and Financial Literacy. Paris, FR: OECD Publishing.

OECD. (2016d). PISA 2015 Technical report. Paris, FR: OECD Publishing.

Pajares, F. (2005). Gender Differences in Mathematics Self-Efficacy Beliefs. In A. M. Gallagher \& J. C. Kaufman (Eds.), Gender differences in mathematics: An integrative psychological approach (pp. 294-315). New York, US: Cambridge University Press.

Pajares, F., \& Miller, M. D. (1994). Role of self-efficacy and self-concept beliefs in mathematical problem solving: A path analysis. Journal of educational psychology, 86(2), 193.

Primi, C., Busdraghi, C., Tomasetto, C., Morsanyi, K., \& Chiesi, F. (2014). Measuring math anxiety in Italian college and high school students: validity, reliability and gender invariance of the Abbreviated Math Anxiety Scale (AMAS). Learning and Individual Differences, 34, $51-56$.

Richardson, M., Abraham, C., \& Bond, R. (2012). Psychological correlates of university stu dents' academic performance: a systematic review and meta-analysis. Psychological bulletin, 138(2), 353. 
Riegle-Crumb, C. (2005). The cross-national context of the gender gap in math and science. In L. V. Hedges \& B. Schneider (Eds.), The social organization of schooling, (pp. 227-243). New York, US: Russell Sage Foundation.

Rohde, T. E., \& Thompson, L. A. (2007). Predicting academic achievement with cognitive ability. Intelligence, 35(1), 83-92.

Ruffing, S., Wach, F. S., Spinath, F. M., Brünken, R., \& Karbach, J. (2015). Learning strategies and general cognitive ability as predictors of gender-specific academic achievement. Frontiers in psychology, 6:1238.

Schultz, T. P. (2002). Why governments should invest more to educate girls. World Development, 30(2), 207-225.

Schunk, D. H., \& Zimmerman, B. J. (1997). Social origins of self-regulatory competence. Educational psychologist, 32(4), 195-208.

Sfard, A. (2005). What Could be More Practical than Good Research?. Educational Studies in Mathematics, 58(3), 393-413.

Spelke, E. S. (2005). Sex differences in intrinsic aptitude for mathematics and science?: a critical review. American Psychologist, 60(9), 950.

Spinath, B., \& Spinath, F. M. (2005). Development of self-perceived ability in elementary school: The role of parents' perceptions, teacher evaluations, and intelligence. Cognitive Development, 20(2), 190-204.

Tiedemann, J. (2000). Parents' gender stereotypes and teachers' beliefs as predictors of children's concept of their mathematical ability in elementary school. Journal of Educational psychology, 92(1), 144

Tomasetto, C. (2013). Matematica per i maschi, italiano per le femmine: Stereotipi di genere e atteggiamenti verso le materie scolastiche tra genitori e figli, IN-MIND ITALIA, 5, 19-24.

Tomasetto, C., Mirisola, A., Galdi, S., \& Cadinu, M. (2015). Parents' math-gender stereotypes, children's self-perception of ability, and children's appraisal of parents' evaluations in 6-year-olds. Contemporary Educational Psychology, 42, 186-198.

Törner, G., \& Arzarello, F. (2013). Grading mathematics education research journals. Mitteilungen der Gesellschaft für Didaktik der Mathematik, 39(95), 31-34.

Virtanen, P., \& Nevgi, A. (2010). Disciplinary and gender differences among higher education students in self-regulated learning strategies. Educational Psychology, 30(3), 323-347.

Weis, M., Heikamp, T., \& Trommsdorff, G. (2013). Gender differences in school achievement: The role of self-regulation. Frontiers in Psychology, 4, 442. Disponibile in http://doi. org/10.3389/fpsyg.2013.00442 (consultato il 28.08.2018).

Winkelmann, H., van den Heuvel-Panhuizen, M., \& Robitzsch, A. (2008). Gender differences in the mathematics achievements of German primary school students: Results from a German large-scale study. ZDM, 40(4), 601-616.

Yee, D. K., \& Eccles, J. S. (1988). Parent perceptions and attributions for children's math achievement. Sex Roles, 19(5), 317-333.

\section{Autore/Chiara Giberti \\ Facoltà di Scienze della Formazione - Libera Università di Bolzano, Italia chiara.gib@gmail.com}

